\title{
On Unique and Nonunique Fixed Points in Metric Spaces and Application to Chemical Sciences
}

\author{
Meena Joshi $\mathbb{D D}^{1}$ and Anita Tomar $\mathbb{D D}^{2}$ \\ ${ }^{1}$ S.G.R.R. (P.G.) College, Dehradun, India \\ ${ }^{2}$ Government Degree College Thatyur (Tehri Garhwal), Uttarakhand, India \\ Correspondence should be addressed to Anita Tomar; anitatmr@yahoo.com
}

Received 1 March 2021; Revised 25 March 2021; Accepted 7 April 2021; Published 7 May 2021

Academic Editor: Calogero Vetro

Copyright (C) 2021 Meena Joshi and Anita Tomar. This is an open access article distributed under the Creative Commons Attribution License, which permits unrestricted use, distribution, and reproduction in any medium, provided the original work is properly cited.

\begin{abstract}
We introduce the notions of a generalized $\Theta$-contraction, a generalized $\Theta_{\mathscr{E}}$-weak contraction, a $\Psi_{\mathscr{E}}$-weak JS-contraction, an integral-type $\Theta_{\mathscr{G}}$-weak contraction, and an integral-type $\Psi_{\mathscr{E}}$-weak JS-contraction to establish the fixed point, fixed ellipse, and fixed elliptic disc theorems. Further, we verify these by illustrative examples with geometric interpretations to demonstrate the authenticity of the postulates. The motivation of this work is the fact that the set of nonunique fixed points may include a geometric figure like a circle, an ellipse, a disc, or an elliptic disc. Towards the end, we provide an application of $\Theta$-contraction to chemical sciences.
\end{abstract}

\section{Introduction and Preliminaries}

The study of the geometry of the set of nonunique fixed points of a map is a significant area of research. There are numerous examples of a map where the set of nonunique fixed points of the self-map includes some geometric shapes. For example, consider a self-map $\mathscr{M}$ on the metric space ( $\mathcal{U}, d)$ with the usual metric defined on the two-dimensional plane $\mathbb{R}^{2}$ as

$$
\mathscr{M}(\mathfrak{u}, \mathfrak{b})=\left(\begin{array}{ll}
(\mathfrak{u}, \mathfrak{v}), & (\mathfrak{u}, \mathfrak{v}) \in \mathfrak{u}^{2}+\mathfrak{v}^{2}=1 \\
(1,0), & \text { otherwise }
\end{array}\right.
$$

Noticeably, the set of nonunique fixed points $\{(\cos n \theta$, $\sin n \theta): n \in \mathbb{Z}, \theta \in[0,2 \pi)\}$ includes the circle $\mathscr{C}((0,0), 1)$ centered at $(0,0)$ having radius 1 ; that is, $\mathscr{C}((0,0), 1)$ is a fixed circle of $\mathscr{M}$. It is significant to mention that there exist maps that map the circle $\mathscr{C}\left(\mathfrak{u}_{0}, \mathfrak{r}\right)$ to itself but do not fix all the points of the circle $\mathscr{C}\left(\mathfrak{u}_{0}, \mathfrak{r}\right)$. For example, let $\mathscr{M}$ be a self-map on the two-dimensional plane $\mathbb{R}^{2}$ defined by

$$
\mathscr{M}(\mathfrak{u}, \mathfrak{v})=\left(\frac{u}{\mathfrak{u t}^{2}+\mathfrak{b}^{2}}, \frac{v}{\mathfrak{t}^{2}+\mathfrak{v}^{2}}\right), \mathfrak{u}, \mathfrak{v} \in \mathbb{R}
$$

Then, $\mathscr{M} \mathscr{C}(0,1)=\mathscr{C}(0,1)$, but map $\mathscr{M}$ fixes only two points $(1,0)$ and $(-1,0)$ of the circle $\mathscr{C}(0,1)$. Noticeably, $\mathscr{M}$ does not fix all the points of $\mathscr{C}(0,1)$. For details on this work, one may refer to [1-23] and the references therein. A geometric figure (a circle, a disc, an ellipse, and so on) included in the set of nonunique fixed points is called a fixed figure (a fixed circle, a fixed disc, a fixed ellipse, and so on) of the self-map [15].

The aim of the present work is to introduce notions of a generalized $\Theta$-contraction, a generalized $\Theta_{\mathscr{E}}$-weak contraction, a $\Psi_{\mathscr{E}}$-weak JS-contraction, a generalized integral-type $\Theta_{\mathscr{G}}$-weak contraction, and an integral-type $\Psi_{\mathscr{G}}$-weak JScontraction to study the geometry of nonunique fixed points. In the sequel, we establish the fixed point, fixed ellipse, and fixed elliptic disc theorems. Further, we verify these by illustrative examples to demonstrate the authenticity of the postulates. Further, we provide an application of Cirić-type $\Theta$ 
-contraction to obtain an application to chemical sciences. To be specific, we solve a boundary value problem arising when a diffusing material is kept in an absorbing medium between parallel walls of specified concentrations.

Definition 1 [24]. A metric on a nonempty set $\mathcal{U}$ is a function $d: \mathscr{U} \times \mathcal{U} \longrightarrow \mathbb{R}^{+}$satisfying

(i) $d(\mathfrak{u}, \mathfrak{v})=0$ iff $\mathfrak{u}=\mathfrak{b}$

(ii) $d(\mathfrak{t}, \mathfrak{v})=d(\mathfrak{v}, \mathfrak{u})$

(iii) $d(\mathfrak{u}, \mathfrak{v}) \leq d(\mathfrak{u}, \mathfrak{w})+d(\mathfrak{w}, \mathfrak{v}), \mathfrak{u}, \mathfrak{v}, \mathfrak{w} \in \mathscr{U}$

Definition 2 [5]. An ellipse having foci at $c_{1}$ and $c_{2}$ in a metric space $(\mathcal{U}, d)$ is defined as

$\mathscr{E}\left(\mathfrak{c}_{1}, \mathfrak{c}_{2}, \mathfrak{a}\right)=\left\{\mathfrak{u} \in \mathscr{U}: d\left(\mathfrak{c}_{1}, \mathfrak{u}\right)+d\left(\mathfrak{c}_{2}, \mathfrak{t}\right)=\mathfrak{a}, \mathfrak{c}_{1}, \mathfrak{c}_{2} \in \mathscr{U}, \mathfrak{a} \in[0, \infty)\right\}$

The midpoint $\mathscr{C}$ of a line $\mathfrak{c}_{1} \mathfrak{c}_{2}$ is known as a center of an ellipse. Here, the segment of length $\mathfrak{a}$ on line $\mathfrak{c}_{1} \mathfrak{c}_{2}$ is the major axis, the line perpendicular to it through the center is the minor axis, and $\mathfrak{a} / 2$ is the length of a semimajor axis of an ellipse. The distance $f=(1 / 2) d\left(\mathfrak{c}_{1}, \mathfrak{c}_{2}\right)$ is the linear eccentricity, and the ratio of linear eccentricity and semimajor axis is the eccentricity; that is, $e=d\left(\mathfrak{c}_{1}, \mathfrak{c}_{2}\right) / \mathfrak{a}$. Visibly, the circles are the ellipses of vanishing eccentricity in which both the focal points are the same; that is, $d\left(\mathfrak{c}_{1}, \mathfrak{c}_{2}\right)=0$. Actually, an ellipse is a compressed circle. Generally, eccentricity is the measure of the deviation of the curve from the circularity of the particular shape.

Example 1. Let $\mathcal{U}=\mathbb{R}$ and a metric $d: \mathcal{U} \times \mathcal{U} \longrightarrow \mathbb{R}^{+}$be defined as $d(\mathfrak{u}, \mathfrak{v})=|\mathfrak{u}-\mathfrak{v}|, \mathfrak{t}, \mathfrak{v} \in \mathscr{U}$; then,

$$
\begin{aligned}
\mathscr{E}(5,10,8) & =\{\mathfrak{u} \in \mathscr{M}: d(5, \mathfrak{u})+d(10, \mathfrak{u})=8\} \\
& =\{\mathfrak{u} \in \mathscr{U}:|5-\mathfrak{u}|+|10-\mathfrak{u}|=8\}=\{3.5,11.5\} .
\end{aligned}
$$

That is, an ellipse centered at 7.5 having foci at 5 and 10 is $\{3.5,11.5\}$.

Definition 3 [25]. Let $\Omega$ symbolize the class of functions $\Theta$ $:(0, \infty) \longrightarrow(1, \infty)$ such that the subsequent conditions hold: $\left(\Theta_{1}\right): \Theta$ is nondecreasing;

$\left(\Theta_{2}\right)$ : for every sequence $\left\{\mathfrak{t}_{n}\right\} \subseteq(0, \infty), \lim _{n \longrightarrow \infty} \Theta \mathfrak{t}_{n}=$ $1 \Leftrightarrow \lim _{n \rightarrow \infty} \mathfrak{t}_{n}=0^{+}$;

$\left(\Theta_{3}\right)$ : there exist $\alpha \in(0,1)$ and $\beta \in(0, \infty]$ such that $\lim _{\mathfrak{u} \longrightarrow 0^{+}}(\Theta(\mathfrak{u})-1) / \mathfrak{u}^{\alpha}=\beta$.

Definition 4 [5]. Let $\mathscr{E}\left(\mathfrak{c}_{1}, \mathfrak{c}_{2}, \mathfrak{a}\right)$ be an ellipse having foci at $\mathfrak{c}_{1}$ and $\mathfrak{c}_{2}$ in a metric space $(\mathcal{U}, d)$. Then, $\mathscr{E}\left(\mathfrak{c}_{1}, \mathfrak{c}_{2}, \mathfrak{a}\right)$ is said to be a fixed ellipse of $\mathscr{M}: \mathscr{U} \longrightarrow \mathscr{U}$ if $\mathscr{M} \mathfrak{t}=\mathfrak{u}, \mathfrak{u} \in \mathscr{E}\left(\mathfrak{c}_{1}, \mathfrak{c}_{2}, \mathfrak{a}\right), \mathfrak{a}$ $\in[0, \infty)$.

\section{Main Results}

In this section, we are dealing with maps satisfying some novel contractions which fix one element of the space or more than one element of the space under suitable conditions and a set of nonunique fixed points, including some geometrical shapes, may be either an ellipse or an elliptic disc. First, we define a generalized $\Theta$-contraction to establish a unique fixed point by giving a short and simple proof.

Definition 5. Let $\Theta:(0, \infty) \longrightarrow(1, \infty) \in \Omega$, and the map $\mathscr{M}$ $: \mathscr{U} \longrightarrow \mathcal{U}$ of a metric space $(\mathcal{U}, d)$ is said to be a generalized $\Theta$-contraction with $\mathfrak{t} \neq \mathfrak{v}$ if

$$
d(\mathscr{M} \mathfrak{t}, \mathscr{M} \mathfrak{v})>0 \Rightarrow \Theta(d(\mathscr{M} \mathfrak{t}, \mathscr{M} \mathfrak{v})) \leq[\Theta(\mathscr{L}(\mathfrak{u}, \mathfrak{v}))]^{\alpha}
$$

where $\mathscr{L}(\mathfrak{u}, \mathfrak{v})=\max \{d(\mathfrak{u}, \mathfrak{v}), \gamma d(\mathfrak{u}, \mathscr{M} \mathfrak{u})+(1-\gamma) d(\mathfrak{v}, \mathscr{M}$ $\mathfrak{v}),(1-\gamma) d(\mathfrak{u}, \mathscr{M} \mathfrak{u})+\gamma d(\mathfrak{v}, \mathscr{M} \mathfrak{v}), \gamma d(\mathfrak{u}, \mathscr{M} \mathfrak{v})+(1-\gamma) d(\mathfrak{v}$, $\mathscr{M} \mathfrak{i t}),(1-\gamma) d(\mathfrak{u}, \mathscr{M} \mathfrak{v})+\gamma d(\mathfrak{v}, \mathscr{M} \mathfrak{i})\}, \gamma \in[0,1), \alpha \in(0,1), \mathfrak{t}$ , $\mathfrak{v} \in \mathscr{U}$.

Remark 6. In the above contraction, if $\gamma=0$, then $\mathscr{M}: \mathscr{U}$ $\longrightarrow \mathcal{U}$ is a Cirić-type $\Theta$-contraction.

Theorem 7. Let $(\mathcal{U}, d)$ be a complete metric space and map $\mathscr{M}: \mathcal{U} \longrightarrow \mathcal{U}$ be a continuous generalized $\Theta$-contraction. Then, $\mathscr{M}$ has a unique fixed point. Also, the sequence of iterates $\left\{\mathscr{M}^{n} \mathfrak{u}\right\}$ converges to a fixed point of $\mathscr{M}$ in $\mathscr{U}$.

Proof. Define a Picard sequence $\left\{\mathfrak{t}_{n}\right\} \subseteq \mathcal{U}, \mathfrak{t}_{n+1}=\mathscr{M} \mathfrak{t}_{n}, n$ $\in \mathbb{N}_{0}$, with initial point $\mathfrak{u}_{0} \in \mathscr{U}$. If for some $n \in \mathbb{N}, \mathscr{M}^{n} \mathfrak{u}=$ $\mathscr{M}^{n+1} \mathfrak{u}$, then $\mathscr{M}^{n} \mathfrak{i t}$ is a fixed point of $\mathscr{M}$ and the proof is complete. So, presume that for each $n, d\left(\mathscr{M}^{n} \mathfrak{t}, \mathscr{M}^{n+1} \mathfrak{u}\right)>0$; then,

$$
\Theta\left(d\left(\mathscr{M}^{n} \mathfrak{u}, \mathscr{M}^{n+1} \mathfrak{u}\right)\right) \leq \Theta\left(\mathscr{L}\left(\mathscr{M}^{n-1} \mathfrak{u}, \mathscr{M}^{n} \mathfrak{u}\right)\right)
$$

where

$$
\begin{aligned}
& \mathscr{L}\left(\mathscr{M}^{n-1} \mathfrak{i t}, \mathscr{M}^{n} \mathfrak{u}\right)=\max \left\{d\left(\mathscr{M}^{n-1} \mathfrak{i t}, \mathscr{M}^{n} \mathfrak{u}\right), \gamma d\left(\mathscr{M}^{n-1} \mathfrak{u t}, \mathscr{M}^{n} \mathfrak{u}\right)\right. \\
& +(1-\gamma) d\left(\mathscr{M}^{n} \mathfrak{i t}, \mathscr{M}^{n+1} \mathfrak{i t}\right),(1-\gamma) d\left(\mathscr{M}^{n-1} \mathfrak{i t}, \mathscr{M}^{n} \mathfrak{i t}\right) \\
& +\gamma d\left(\mathscr{M}^{n} \mathfrak{u}, \mathscr{M}^{n+1} \mathfrak{u}\right), \gamma d\left(\mathscr{M}^{n-1} \mathfrak{u}, \mathscr{M}^{n+1} \mathfrak{u}\right) \\
& +(1-\gamma) d\left(\mathscr{M}^{n} \mathfrak{u}, \mathscr{M}^{n} \mathfrak{u}\right),(1-\gamma) d\left(\mathscr{M}^{n-1} \mathfrak{u}, \mathscr{M}^{n+1} \mathfrak{u}\right) \\
& \left.+\gamma d\left(\mathscr{M}^{n} \mathfrak{u t}, \mathscr{M}^{n} \mathfrak{u}\right)\right\} \\
& =\max \left\{d\left(\mathscr{M}^{n-1} \mathfrak{u}, \mathscr{M}^{n} \mathfrak{i t}\right), \gamma d\left(\mathscr{M}^{n-1} \mathfrak{u}, \mathscr{M}^{n} \mathfrak{i t}\right)\right. \\
& +(1-\gamma) d\left(\mathscr{M}^{n} \mathfrak{u}, \mathscr{M}^{n+1} \mathfrak{i}\right),(1-\gamma) d\left(\mathscr{M}^{n-1} \mathfrak{u}, \mathscr{M}^{n} \mathfrak{u}\right) \\
& +\gamma d\left(\mathscr{M}^{n} \mathfrak{i t}, \mathscr{M}^{n+1} \mathfrak{i}\right), \gamma d\left(\mathscr{M}^{n-1} \mathfrak{u}, \mathscr{M}^{n+1} \mathfrak{u}\right), \\
& \cdot(1-\gamma) d\left(\mathscr{M}^{n-1} \mathfrak{i t}, \mathscr{M}^{n+1} \mathfrak{i t}\right\}
\end{aligned}
$$

Case 1. If $d\left(\mathscr{M}^{n-1} \mathfrak{i}, \mathscr{M}^{n} \mathfrak{u}\right) \leq d\left(\mathscr{M}^{n} \mathfrak{i t}, \mathscr{M}^{n+1} \mathfrak{u}\right)$, then

$$
\mathscr{L}\left(\mathscr{M}^{n-1} \mathfrak{u}, \mathscr{M}^{n} \mathfrak{u}\right)=d\left(\mathscr{M}^{n} \mathfrak{u}, \mathscr{M}^{n+1} \mathfrak{u}\right)
$$

That is, $\Theta\left(d\left(\mathscr{M}^{n} \mathfrak{u}, \mathscr{M}^{n+1} \mathfrak{u}\right)\right) \leq\left[\Theta\left(d\left(\mathscr{M}^{n} \mathfrak{u}, \mathscr{M}^{n+1} \mathfrak{u}\right)\right)\right]^{\alpha}$, $\alpha \in(0,1)$, a contradiction. 
Case 2. If $d\left(\mathscr{M}^{n-1} \mathfrak{i}, \mathscr{M}^{n} \mathfrak{u}\right) \geq d\left(\mathscr{M}^{n} \mathfrak{u}, \mathscr{M}^{n+1} \mathfrak{u}\right)$, then

$$
\mathscr{L}\left(\mathscr{M}^{n-1} \mathfrak{i t}, \mathscr{M}^{n} \mathfrak{t}\right)=d\left(\mathscr{M}^{n-1} \mathfrak{u}, \mathscr{M}^{n} \mathfrak{i t}\right)
$$

That is, $\Theta\left(d\left(\mathscr{M}^{n} \mathfrak{t}, \mathscr{M}^{n+1} \mathfrak{t}\right)\right) \leq\left[\Theta\left(d\left(\mathscr{M}^{n-1} \mathfrak{t}, \mathscr{M}^{n} \mathfrak{u}\right)\right)\right]^{\alpha}$.

Following a similar pattern,

$$
\begin{aligned}
\Theta\left(d\left(\mathscr{M}^{n} \mathfrak{u}, \mathscr{M}^{n+1} \mathfrak{i}\right)\right) & \leq\left[\Theta\left(d\left(\mathscr{M}^{n-1} \mathfrak{i}, \mathscr{M}^{n} \mathfrak{t}\right)\right)\right]^{\alpha} \ldots \\
& \leq\left[\Theta\left(d\left(\mathfrak{u}, \mathscr{M}_{\mathfrak{u}}\right)\right)\right]^{\alpha^{n}} \longrightarrow 1, \text { as } n \longrightarrow \infty
\end{aligned}
$$

Using $\left(\Theta_{2}\right), \lim _{n \longrightarrow \infty} d(\mathfrak{u}, \mathscr{M} \mathfrak{u})=0$.

Using $\left(\Theta_{3}\right)$, there exist $\beta \in(0, \infty)$ such that $\lim _{n-}$ $\left.\left(d\left(\mathscr{M}^{n} \mathfrak{t}, \mathscr{M}^{n+1} \mathfrak{u}\right)\right)-1\right) /\left(d\left(\mathscr{M}^{n} \mathfrak{i t}, \mathscr{M}^{n+1} \mathfrak{i t}\right)\right)^{\alpha}=\beta$.

If $\beta \in(0, \infty)$, then for $\varepsilon_{1}=\beta / 4>0$, there exists $N_{1}>0$ such that

$$
\begin{aligned}
& \left|\frac{\Theta\left(d\left(\mathscr{M}^{n} \mathfrak{u}, \mathscr{M}^{n+1} \mathfrak{i}\right)\right)-1}{\left(d\left(\mathscr{M}^{n} \mathfrak{u}, \mathscr{M}^{n+1} \mathfrak{u}\right)\right)^{\alpha}}-\beta\right|<\varepsilon, n \geq N_{1}, \\
& \Rightarrow \frac{\Theta\left(d\left(\mathscr{M}^{n} \mathfrak{u}, \mathscr{M}^{n+1} \mathfrak{u}\right)\right)-1}{\left(d\left(\mathscr{M}^{n} \mathfrak{u}, \mathscr{M}^{n+1} \mathfrak{u}\right)\right)^{\alpha}}>\beta-\varepsilon_{1} \\
& \quad=\frac{3}{4} \beta>\varepsilon_{1}, n \geq N_{1} .
\end{aligned}
$$

That is, $\left(d\left(\mathscr{M}^{n} \mathfrak{u}, \mathscr{M}^{n+1} \mathfrak{u}\right)\right)^{\alpha}<\left(1 / \varepsilon_{1}\right)\left(\Theta\left(d\left(\mathscr{M}^{n} \mathfrak{u}, \mathscr{M}^{n+1} \mathfrak{u}\right.\right.\right.$ )) -1$), n \geq N_{1}$.

If $\beta=\infty$, then for any $\varepsilon_{2}>0$, there exists $N_{2}>0$ such that

$$
\frac{\Theta\left(d\left(\mathscr{M}^{n} \mathfrak{u}, \mathscr{M}^{n+1} \mathfrak{u}\right)\right)-1}{\left(d\left(\mathscr{M}^{n} \mathfrak{u}, \mathscr{M}^{n+1} \mathfrak{u}\right)\right)^{\alpha}}>\varepsilon_{2}, n \geq N_{2} .
$$

That is, $\left(d\left(\mathscr{M}^{n} \mathfrak{i t}, \mathscr{M}^{n+1} \mathfrak{i t}\right)\right)^{\alpha}<\left(1 / \varepsilon_{2}\right)\left(\Theta\left(d\left(\mathscr{M}^{n} \mathfrak{t}, \mathscr{M}^{n+1} \mathfrak{u}\right.\right.\right.$ )) -1$), n>N_{2}$.

Thus, for all $\beta \in(0, \infty]$ and $\mu=\max \left\{1 / \varepsilon_{1}, 1 / \varepsilon_{2}\right\}$, there exists $N=\max \left\{N_{1}, N_{2}\right\}$ such that

$$
\begin{aligned}
& \left(d\left(\mathscr{M}^{n} \mathfrak{u}, \mathscr{M}^{n+1} \mathfrak{u}\right)\right)^{\alpha}<\mu\left(\Theta\left(d\left(\mathscr{M}^{n} \mathfrak{u}, \mathscr{M}^{n+1} \mathfrak{u}\right)\right)-1\right), n>N \\
& \quad \leq \mu[\Theta(d(\mathfrak{u}, \mathscr{M} \mathfrak{u}))]-1 \longrightarrow 0, \text { as } n \longrightarrow \infty\left(\mathrm{using}\left(\Theta_{2}\right)\right) .
\end{aligned}
$$

That is, $\lim _{n \longrightarrow \infty}\left(d\left(\mathscr{M}^{n} \mathfrak{i}, \mathscr{M}^{n+1} \mathfrak{t}\right)\right)^{\alpha}=0$ implies that there exists $n \geq N$ such that

$$
d\left(\mathscr{M}^{n} \mathfrak{u}, \mathscr{M}^{n+1} \mathfrak{i t}\right) \leq \frac{1}{n^{1 / \alpha}}, n \geq N
$$

If $n>m$,

$$
\begin{aligned}
d\left(\mathscr{M}^{m} \mathfrak{u}, \mathscr{M}^{n} \mathfrak{u}\right) \leq & d\left(\mathscr{M}^{m} \mathfrak{u}, \mathscr{M}^{m+1} \mathfrak{u}\right)+d\left(\mathscr{M}^{m+1} \mathfrak{u}, \mathscr{M}^{m+2} \mathfrak{u}\right) \\
& +\cdots+d\left(\mathscr{M}^{n-1} \mathfrak{u}, \mathscr{M}^{n} \mathfrak{u}\right) \\
\leq & \frac{1}{m^{1 / \alpha}}+\frac{1}{(m+1)^{1 / \alpha}}+\cdots+\frac{1}{(n-1)^{1 / \alpha}} \\
\leq & \sum_{i=n}^{\infty} \frac{1}{i^{1 / \alpha}} .
\end{aligned}
$$

Since $\alpha \in(0,1)$, series $\sum_{i=n}^{\infty} 1 / i^{1 / \alpha}$ is convergent and $\lim _{n, m \longrightarrow \infty} d\left(\mathscr{M}^{m} \mathfrak{t}, \mathscr{M}^{n} \mathfrak{u}\right)$ exists and is finite; that is, $\left\{\mathscr{M}^{n} \mathfrak{u}\right.$ \} is a Cauchy sequence.

Since $\mathcal{U}$ is complete, $\left\{\mathscr{M}^{n} \mathfrak{u}\right\}$ converges to $\mathfrak{t}^{*} \in \mathcal{U}$. Since $\mathscr{M}$ is continuous, $\left\{\mathscr{M}^{n} \mathfrak{i}\right\} \longrightarrow \mathfrak{t}^{*} \Rightarrow\left\{\mathscr{M}^{n+1} \mathfrak{u}\right\} \longrightarrow \mathscr{M}_{\mathfrak{t}}{ }^{*}$. By definition of limit $\mathscr{M} \mathfrak{t}^{*}=\mathfrak{u}^{*}$, that is, $\mathfrak{u}^{*}$ is a fixed point of $\mathscr{M}$.

Let $\mathfrak{w}^{*}$ be another fixed point of $\mathscr{U}$. So $d\left(\mathscr{M} \mathfrak{u}^{*}, \mathscr{M} \mathfrak{w}^{*}\right)$ $=d\left(\mathfrak{t}^{*}, \mathfrak{w}^{*}\right)>0$. Now,

$$
\Theta\left(d\left(\mathscr{M} \mathfrak{t}^{*}, \mathscr{M} \mathfrak{w}^{*}\right)\right) \leq\left[\Theta\left(\mathscr{L}\left(\mathfrak{u}^{*}, \mathfrak{w}^{*}\right)\right)\right]^{\alpha},
$$

where

$$
\begin{aligned}
& \mathscr{L}\left(\mathfrak{u t}^{*}, \mathfrak{w}^{*}\right)=\max \left\{d\left(\mathfrak{u t}^{*}, \mathfrak{w}^{*}\right), \gamma d\left(\mathfrak{u}^{*}, \mathscr{M} \mathfrak{t}^{*}\right)+(1-\gamma) d\left(\mathfrak{w}^{*}, \mathscr{M} \mathfrak{w}^{*}\right),\right. \\
& \cdot(1-\gamma) d\left(\mathfrak{u t}^{*}, M_{\mathfrak{u}} \mathfrak{t}^{*}\right)+\gamma d\left(\mathfrak{w}^{*}, \mathscr{M} \mathfrak{w}^{*}\right), \gamma d\left(\mathfrak{u}^{*}, \mathscr{M} \mathfrak{w}^{*}\right) \\
& \left.+(1-\gamma) d\left(\mathfrak{w}^{*}, \mathscr{M} \mathfrak{u}^{*}\right),(1-\gamma) d\left(\mathfrak{t}^{*}, \mathscr{M} \mathfrak{w}^{*}\right)+\gamma d\left(\mathfrak{w}^{*}, \mathscr{M} \mathfrak{u}^{*}\right)\right\} \\
& =\max \left\{d\left(\mathfrak{u}^{*}, \mathfrak{w}^{*}\right), \gamma d\left(\mathfrak{u}^{*}, \mathfrak{u}^{*}\right)+(1-\gamma) d\left(\mathfrak{w}^{*}, \mathfrak{w}^{*}\right),(1-\gamma) d\left(\mathfrak{u}^{*}, \mathfrak{u}^{*}\right)\right. \\
& +\gamma d\left(\mathfrak{w}^{*}, \mathfrak{w}^{*}\right), \gamma d\left(\mathfrak{u}^{*}, \mathfrak{w}^{*}\right)+(1-\gamma) d\left(\mathfrak{w}^{*}, \mathfrak{u}^{*}\right),(1-\gamma) d\left(\mathfrak{u}^{*}, \mathfrak{w}^{*}\right) \\
& \left.+\gamma d\left(\mathfrak{w}^{*}, \mathfrak{t}^{*}\right)\right\} \\
& =\max \left\{d\left(\mathfrak{u}^{*}, \mathfrak{w}^{*}\right), \gamma d\left(\mathfrak{u}^{*}, \mathfrak{w}^{*}\right)+(1-\gamma) d\left(\mathfrak{w}^{*}, \mathfrak{t}^{*}\right),(1-\gamma) d\left(\mathfrak{u}^{*}, \mathfrak{w}^{*}\right)\right. \\
& \left.+\gamma d\left(\mathfrak{w}^{*}, \mathfrak{u}^{*}\right)\right\}=d\left(\mathfrak{u}^{*}, \mathfrak{w}^{*}\right) \text {. }
\end{aligned}
$$

That is, $\Theta\left(d\left(\mathscr{M} \mathfrak{t}^{*}, \mathscr{M} \mathfrak{w}^{*}\right)\right) \leq\left[\Theta\left(d\left(\mathfrak{t}^{*}, \mathfrak{w}^{*}\right)\right)\right]^{\alpha} \leq \Theta(d($ $\left.\left.\mathfrak{u}^{*}, \mathfrak{w}^{*}\right)\right)$.

That is, $\Theta\left(d\left(\mathfrak{t}^{*}, \mathfrak{w}^{*}\right)\right) \leq \Theta\left(d\left(\mathfrak{u}^{*}, \mathfrak{w}^{*}\right)\right)$, a contradiction.

Hence, $\mathscr{M}$ has a unique fixed point in $\mathscr{U}$.

Theorem 8. Let $(\mathcal{U}, d)$ be a complete metric space and map $\mathscr{M}: \mathcal{U} \longrightarrow \mathcal{U}$ be a continuous Cirić-type $\Theta$-contraction. Then, $\mathscr{M}$ has a unique fixed point. Also, the sequence of iterates $\left\{\mathscr{M}^{n} \mathfrak{u}\right\}$ converges to a fixed point of $\mathscr{M}$ in $\mathcal{U}$.

Proof. The proof follows the pattern of Theorem 7 on taking $\gamma=0$.

The subsequent example appreciates that Theorem 8 gives assurance of the uniqueness of the fixed point.

Example 2. Let $\mathcal{U}=\left\{\mathfrak{u}_{n}=2 n-1: n \in \mathbb{N}\right\}$ and a metric $d$ $: \mathscr{U} \times \mathscr{U} \longrightarrow \mathbb{R}^{+}$be defined as $d(\mathfrak{u}, \mathfrak{v})=|\mathfrak{u}-\mathfrak{v}|$. Then, $(\mathcal{U}, d$ ) is a complete metric space.

Let $\Theta(t)=e^{t e^{t}} \in \Omega, \gamma=0$. 
Define a self-map $\mathscr{M}: \mathscr{U} \longrightarrow \mathscr{U}$ as

$$
\mathscr{M} \mathfrak{u}=\left(\begin{array}{ll}
\mathfrak{u}_{1}, & \mathfrak{u}=\mathfrak{t}_{1}, \\
\mathfrak{u}_{n-1}, & \mathfrak{u}=\mathfrak{t}_{n}, n \geq 2 .
\end{array}\right.
$$

Then,

$$
\begin{aligned}
\mathscr{L}\left(\mathfrak{u}_{n}, \mathfrak{i t}_{1}\right) & =\max \left\{d\left(\mathfrak{u}_{n}, \mathfrak{u}_{1}\right), d\left(\mathfrak{u}_{n}, \mathscr{M} \mathfrak{u}_{n}\right), d\left(\mathfrak{u}_{1}, \mathscr{M} \mathfrak{u}_{1}\right), d\left(\mathfrak{u}_{1}, \mathscr{M} \mathbf{u}_{n}\right), d\left(\mathfrak{u}_{n}, \mathscr{M} \mathfrak{u}_{1}\right)\right\} \\
& =\max \{d(2 n-1,1), d(2 n-1,2 n-3), 0, d(1,2 n-3), d(2 n-1,1)\} \\
& =\max \{|2 n-2|,|2|,|2 n-4|\}=2 n-2, n \geq 2 .
\end{aligned}
$$

Now,

$$
\begin{aligned}
& \lim _{n \rightarrow \infty} \frac{d\left(\mathscr{M} \mathfrak{u}_{n}, \mathscr{M} \mathfrak{u}_{1}\right)}{\mathscr{L}\left(\mathfrak{u}_{n}, \mathfrak{u t}_{1}\right)} \\
& \quad=\lim _{n \rightarrow \infty} \frac{\left|\mathscr{M} \mathfrak{u}_{n}-\mathscr{M} \mathfrak{u}_{1}\right|}{\mathscr{L}\left(\mathfrak{u}_{n}, \mathfrak{u}_{1}\right)} \\
& \quad=\lim _{n \rightarrow \infty} \frac{|2 n-3-1|}{2 n-2}=1, n \geq 2 .
\end{aligned}
$$

Clearly, $\mathscr{M}$ is neither a Ćirić-type contraction [26] nor a Banach contraction [27].

Now, we claim that $\mathscr{M}$ satisfies Ćirić-type $\Theta$-contraction; that is,

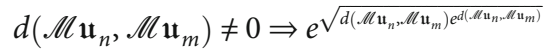

$$
\begin{aligned}
& \leq e^{\alpha \sqrt{d\left(\mathfrak{u}_{n}, \mathfrak{u}_{m}\right) e^{d\left(\mathfrak{u}_{n}, \mathbf{u}_{m}\right)}}}, \alpha \in(0,1) \\
& \Rightarrow d\left(\mathscr{M} \mathfrak{u}_{n}, \mathscr{M} \mathfrak{u}_{m}\right) e^{d\left(\mathscr{M} \mathfrak{u}_{n}, \mathscr{M} \mathfrak{u}_{m}\right)} \\
& \leq \alpha^{2}\left[d\left(\mathfrak{u}_{n}, \mathfrak{u}_{m}\right) e^{d\left(\mathfrak{u}_{n}, \mathfrak{u}_{m}\right)}\right], \alpha \in(0,1) \\
& \Rightarrow \frac{d\left(\mathscr{M} \mathfrak{u}_{n}, \mathscr{M} \mathfrak{u}_{m}\right) e^{d\left(\mathscr{M} \mathfrak{u}_{n}, \mathscr{M} \mathfrak{u}_{m}\right)}}{d\left(\mathfrak{u}_{n}, \mathfrak{t}_{m}\right) e^{d\left(\mathfrak{u}_{n}, \mathfrak{u}_{m}\right)}} \leq \alpha^{2}, \alpha \in(0,1) .
\end{aligned}
$$

Case 1. When $n=1$ and $m \geq 2$,

$$
\begin{aligned}
& \frac{d\left(\mathscr{M} \mathfrak{t}_{n}, \mathscr{M} \mathfrak{u}_{m}\right) e^{d\left(\mathscr{M} \mathfrak{u}_{n}, \mathscr{M} \mathfrak{u}_{m}\right)}}{d\left(\mathfrak{u}_{n}, \mathfrak{u}_{m}\right) e^{d\left(\mathfrak{u}_{n}, \mathfrak{u}_{m}\right)}} \\
& \quad=\frac{|4-2 m| e^{|4-2 m|}}{|2-2 m| e^{|2-2 m|}}=\frac{(2 m-4) e^{(2 m-4)}}{(2 m-2) e^{(2 m-2)}} \leq e^{-2} .
\end{aligned}
$$

Case 2. When $n>m>1$,

$$
\begin{aligned}
& \frac{d\left(\mathscr{M}_{\mathfrak{u}_{n}}, \mathscr{M} \mathfrak{u}_{m}\right) e^{d\left(\mathscr{M} \mathfrak{u}_{n}, \mathscr{M}_{\mathfrak{u}_{m}}\right)}}{d\left(\mathfrak{u}_{n}, \mathfrak{u}_{m}\right) e^{d\left(\mathfrak{u}_{n}, \mathfrak{u}_{m}\right)}} \\
& =\frac{|2 n-2 m-6| e^{|2 n-2 m-6|}}{|2 n-2 m-2| e^{|2 n-2 m-2|}} \\
& =\frac{(2 n-2 m-6) e^{(2 n-2 m-6)}}{(2 n-2 m-2) e^{(2 n-2 m-2)}} \leq e^{-4} .
\end{aligned}
$$

Thus, $\mathscr{M}$ is a Ćirić-type $\Theta$-contraction with $\alpha=\max \{$ $\left.e^{-4}, e^{-2}\right\}=e^{-2}$ and has a unique fixed point 1. Further, $\lim _{n \rightarrow \infty} \mathscr{M}^{n} \mathfrak{t}_{1}=1$.

Remark 9. Theorems 7 and 8 are improvements, extensions, and generalizations of Banach [27], Ćirić [26], Jleli and Samet [25], and references therein. Further, on taking $\Theta(t)=e^{t}, t$ $>0$, in these results, we obtain some novel results which are generalizations of existing results in the literature.

Next, following Joshi et al. [5], we define an elliptic disc and a fixed elliptic disc to study the geometry of nonunique fixed points in a metric space.

Definition 10. An elliptic disc having foci at $\mathfrak{c}_{1}$ and $\mathfrak{c}_{2}$ in a metric space $(\mathcal{U}, d)$ is defined as $\mathscr{E}_{\mathscr{D}}\left(\mathfrak{c}_{1}, \mathfrak{c}_{2}, \mathfrak{a}\right)=\{\mathfrak{u} \in \mathscr{U}: d($ $\left.\left.\mathfrak{c}_{1}, \mathfrak{u}\right)+d\left(\mathfrak{c}_{2}, \mathfrak{u}\right) \leq \mathfrak{a}, \mathfrak{c}_{1}, \mathfrak{c}_{2} \in \mathscr{M}, \mathfrak{a} \in[0, \infty)\right\}$.

Remark 11. For defining an ellipse or elliptic disc $\mathfrak{a} \geq d\left(\mathfrak{c}_{1}, \mathfrak{c}_{2}\right)$

Example 3. Let $\mathcal{U}=\mathbb{R}^{2}$ and a metric $d: \mathcal{U} \times \mathcal{U} \longrightarrow \mathbb{R}^{+}$be defined as $d(\mathfrak{u}, \mathfrak{b})=\sqrt{\left(\mathfrak{u}_{1}-\mathfrak{v}_{1}\right)^{2}+\left(\mathfrak{t}_{2}-\mathfrak{b}_{2}\right)^{2}}, \mathfrak{u} \ll\left(\mathfrak{u}_{1}, \mathfrak{u}_{2}\right)$ , $\mathfrak{v} \ll\left(\mathfrak{v}_{1}, \mathfrak{v}_{2}\right) \in \mathcal{U}$; then,

$$
\begin{aligned}
\mathscr{E}_{\mathscr{D}}((-5,0),(5,0), 12)= & \left\{\mathfrak{u} \in \mathscr{U}: d\left((-5,0),\left(\mathfrak{u}_{1}, \mathfrak{u}_{2}\right)\right)\right. \\
& \left.+d\left((5,0),\left(\mathfrak{u}_{1}, \mathfrak{u}_{2}\right)\right) \leq 12\right\} \\
= & \left\{\mathfrak{u} \in \mathscr{U}: \sqrt{\left(\mathfrak{u}_{1}+5\right)^{2}+\mathfrak{u}_{2}^{2}}\right. \\
& \left.+\sqrt{\left(\mathfrak{u}_{1}-5\right)^{2}+\mathfrak{t}_{2}^{2}} \leq 12\right\},
\end{aligned}
$$

which is shown by the blue shaded portion in Figure 1.

Definition 12. Let $\mathscr{E}_{\mathscr{D}}\left(\mathfrak{c}_{1}, \mathfrak{c}_{2}, \mathfrak{a}\right)$ be an elliptic disc having foci at $\mathfrak{c}_{1}$ and $\mathfrak{c}_{2}$ in a metric space $(\mathcal{U}, d)$. Then, $\mathscr{E}_{\mathscr{D}}\left(\mathfrak{c}_{1}, \mathfrak{c}_{2}, \mathfrak{a}\right)$ is said to be a fixed elliptic disc of map $\mathscr{M}: \mathscr{U} \longrightarrow \mathscr{U}$ if $\mathscr{M} \mathfrak{t}$ $=\mathfrak{t}, \mathfrak{t} \in \mathscr{E}_{\mathscr{D}}\left(\mathfrak{c}_{1}, \mathfrak{c}_{2}, \mathfrak{a}\right), \mathfrak{a} \in[0, \infty)$.

We now introduce and exploit a generalized $\Theta_{\mathscr{C}}$-weak contraction to demonstrate that the set of nonunique fixed points of a map includes an ellipse or an elliptic disc.

Definition 13. Let $\Theta:(0, \infty) \longrightarrow(1, \infty)$ be an increasing function. A map $\mathscr{M}: \mathscr{U} \longrightarrow \mathscr{U}$ of a metric space $(\mathcal{U}, d)$ is said to be a generalized $\Theta_{\mathscr{E}}$-weak contraction with $\mathfrak{u} \neq \mathfrak{b}$, if

$$
d(\mathfrak{u}, \mathscr{M} \mathfrak{u})>0 \Rightarrow \Theta(d(\mathfrak{u}, \mathscr{M} \mathfrak{u})) \leq[\Theta(\mathscr{L}(\mathfrak{u}, \mathfrak{b}))]^{\alpha}
$$

where $\mathscr{L}(\mathfrak{u}, \mathfrak{v})=\max \{d(\mathfrak{u}, \mathfrak{v}), \gamma d(\mathfrak{u}, \mathscr{M} \mathfrak{i})+(1-\gamma) d(\mathfrak{v}, \mathscr{M}$ $\mathfrak{v}),(1-\gamma) d(\mathfrak{u}, \mathscr{M} \mathfrak{u})+\gamma d(\mathfrak{v}, \mathscr{M} \mathfrak{v}), \gamma d(\mathfrak{u}, \mathscr{M} \mathfrak{v})+(1-\gamma) d(\mathfrak{v}$, $\mathscr{M} \mathfrak{u}),(1-\gamma) d(\mathfrak{u}, \mathscr{M} \mathfrak{v})+\gamma d(\mathfrak{v}, \mathscr{M} \mathfrak{u})\}, \gamma \in[0,1), \alpha \in(0,1), \mathfrak{u}$ , $\mathfrak{v} \in \mathscr{U}$.

Remark 14. In the above contraction, if $\gamma=0$, then $\mathscr{M}: \mathscr{U}$ $\longrightarrow \mathcal{U}$ is said to be a Ćirić-type $\Theta_{\mathscr{E}}$-weak contraction. 


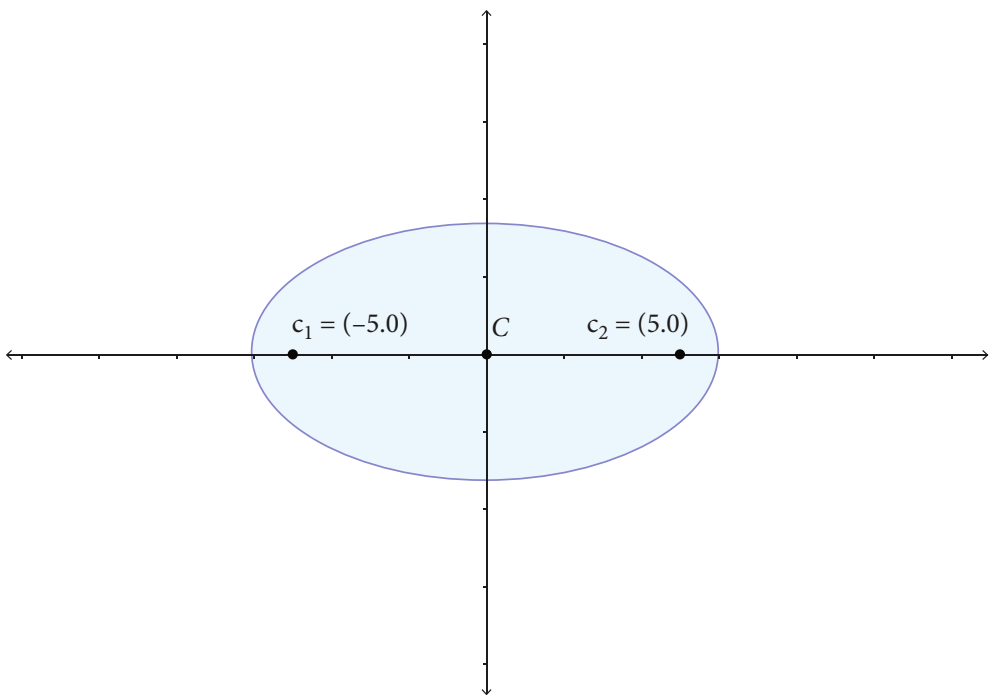

Figure 1: The elliptic disc $\mathscr{E}_{\mathscr{D}}((-5,0),(5,0), 12)$ centered at $(0,0)$ having foci at $(-5,0)$ and $(5,0)$ is shown in this figure.

Theorem 15. Let $\mathscr{E}\left(\mathfrak{c}_{1}, \mathfrak{c}_{2}, \mathfrak{a}\right)$ be an ellipse in a metric space $(\mathcal{U}, d)$ and $\mathfrak{a}=(1 / 2)\{\inf d(\mathfrak{i}, \mathscr{M} \mathfrak{i}): \mathfrak{i t} \neq \mathscr{M} \mathfrak{t}\}$. If map $\mathscr{M}$ $: \mathcal{U} \longrightarrow \mathcal{U}$ is a generalized $\Theta_{\mathscr{E}}$-weak contraction with $\mathfrak{c}_{1}, \mathfrak{c}_{2}$ $\in \mathscr{U}$ and $d\left(\mathfrak{c}_{1}, \mathscr{M} \mathfrak{u}\right)+d\left(\mathfrak{c}_{2}, \mathscr{M} \mathfrak{u}\right)=\mathfrak{a}, \mathfrak{t} \in \mathscr{E}\left(\mathfrak{c}_{1}, \mathfrak{c}_{2}, \mathfrak{a}\right)$, then $\mathscr{E}\left(\mathfrak{c}_{1}, \mathfrak{c}_{2}, \mathfrak{a}\right)$ is a fixed ellipse of $\mathscr{M}$.

Proof. Let $\mathfrak{u} \in \mathscr{E}\left(\mathfrak{c}_{1}, \mathfrak{c}_{2}, \mathfrak{a}\right)$ be any arbitrary point and $\mathscr{M} \mathfrak{u} \neq \mathfrak{u}$ . From the definition of $\mathfrak{a}, d(\mathfrak{u}, \mathscr{M} \mathfrak{u}) \geq 2 \mathfrak{a}$, suppose $\mathscr{M} \mathfrak{c}_{1} \neq \mathfrak{c}_{1}$ and $\mathscr{M} \mathfrak{c}_{2} \neq \mathfrak{c}_{2}$, so we have $\left.\left.d\left(\mathfrak{c}_{1}, \mathscr{M}_{\mathfrak{c}_{1}}\right)\right)>0, d\left(\mathfrak{c}_{2}, \mathscr{M} \mathfrak{c}_{2}\right)\right)>0$, and

$$
\begin{aligned}
\Theta\left(d\left(\mathfrak{c}_{1}, \mathscr{M} \mathfrak{c}_{1}\right)\right) \leq & {\left[\Theta\left(\mathscr{L}\left(\mathfrak{c}_{1}, \mathfrak{c}_{1}\right)\right)\right]^{\alpha} } \\
= & {\left[\Theta \left(\operatorname { m a x } \left\{d\left(\mathfrak{c}_{1}, \mathfrak{c}_{1}\right), \gamma d\left(\mathfrak{c}_{1}, \mathscr{M} \mathfrak{c}_{1}\right)\right.\right.\right.} \\
& +(1-\gamma) d\left(\mathfrak{c}_{1}, \mathscr{M} \mathfrak{c}_{1}\right),(1-\gamma) d\left(\mathfrak{c}_{1}, \mathscr{M} \mathfrak{c}_{1}\right) \\
& +\gamma d\left(\mathfrak{c}_{1}, \mathscr{M} \mathfrak{c}_{1}\right) \gamma d\left(\mathfrak{c}_{1}, \mathscr{M} \mathfrak{c}_{1}\right) \\
& +(1-\gamma) d\left(\mathfrak{c}_{1}, \mathscr{M} \mathfrak{c}_{1}\right),(1-\gamma) d\left(\mathfrak{c}_{1}, \mathscr{M} \mathfrak{c}_{1}\right) \\
& \left.\left.\left.+\gamma d\left(\mathfrak{c}_{1}, \mathscr{M} \mathfrak{c}_{1}\right)\right\}\right)\right]^{\alpha} \\
= & {\left[\Theta\left(\max \left\{0, d\left(\mathfrak{c}_{1}, \mathscr{M} \mathfrak{c}_{1}\right)\right\}\right)\right]^{\alpha}=\left[\Theta\left(d\left(\mathfrak{c}_{1}, \mathscr{M} \mathfrak{c}_{1}\right)\right)\right]^{\alpha} } \\
< & \Theta\left(d\left(\mathfrak{c}_{1}, \mathscr{M} \mathfrak{c}_{1}\right)\right), \alpha \in(0,1), \text { a contradiction. }
\end{aligned}
$$

So $\mathscr{M} \mathfrak{c}_{1}=\mathfrak{c}_{1}$. Similarly, $\mathscr{M} \mathfrak{c}_{2}=\mathfrak{c}_{2}$.

Again, since $d(\mathfrak{t}, \mathscr{M} \mathfrak{t})>0$,

$$
\begin{aligned}
\Theta(d(\mathfrak{t}, \mathscr{M} \mathfrak{u})) \leq & {\left[\Theta\left(\mathscr{L}\left(\mathfrak{u}, \mathfrak{c}_{1}\right)\right)\right]^{\alpha} } \\
= & {\left[\Theta \left(\operatorname { m a x } \left\{d\left(\mathfrak{u}, \mathfrak{c}_{1}\right), \gamma d(\mathfrak{u}, \mathscr{M} \mathfrak{u})\right.\right.\right.} \\
& +(1-\gamma) d\left(\mathfrak{c}_{1}, \mathscr{M} \mathfrak{c}_{1}\right),(1-\gamma) d(\mathfrak{u}, \mathscr{M} \mathfrak{u}) \\
& +\gamma d\left(\mathfrak{c}_{1}, \mathscr{M} \mathfrak{c}_{1}\right) \gamma d\left(\mathfrak{u}, \mathscr{M} \mathfrak{c}_{1}\right) \\
& \left.\left.\left.+(1-\gamma) d\left(\mathfrak{c}_{1}, \mathscr{M} \mathfrak{u}\right),(1-\gamma) d\left(\mathfrak{u}, \mathscr{M} \mathfrak{c}_{1}\right)+\gamma d\left(\mathfrak{c}_{1}, \mathscr{M} \mathfrak{u}\right)\right\}\right)\right]^{\alpha} \\
< & {\left[\Theta \left(\operatorname { m a x } \left\{2 \mathfrak{a}, \gamma d(\mathfrak{u}, \mathscr{M} \mathfrak{u}),(1-\gamma) d(\mathfrak{u}, \mathscr{M} \mathfrak{u}), \gamma d\left(\mathfrak{u}, \mathfrak{c}_{1}\right)\right.\right.\right.} \\
& \left.\left.\left.+(1-\gamma) d\left(\mathfrak{c}_{1}, \mathscr{M} \mathfrak{u}\right),(1-\gamma) d\left(\mathfrak{u}, \mathfrak{c}_{1}\right)+\gamma d\left(\mathfrak{c}_{1}, \mathscr{M} \mathfrak{u}\right)\right\}\right)\right]^{\alpha} \\
< & \Theta\left(\operatorname { m a x } \left\{2 \mathfrak{a}, \gamma d(\mathfrak{u}, \mathscr{M} \mathfrak{u}),(1-\gamma) d(\mathfrak{u}, \mathscr{M} \mathfrak{u}), \gamma d\left(\mathfrak{u}, \mathfrak{c}_{1}\right)\right.\right. \\
+ & \left.\left.(1-\gamma) d\left(\mathfrak{c}_{1}, \mathscr{M} \mathfrak{u}\right),(1-\gamma) d\left(\mathfrak{u}, \mathfrak{c}_{1}\right)+\gamma d\left(\mathfrak{c}_{1}, \mathscr{M} \mathfrak{u}\right)\right\}\right), \alpha \in(0,1) .
\end{aligned}
$$

Case 1. If $\max \{2 \mathfrak{a}, \gamma d(\mathfrak{u}, \mathscr{M} \mathfrak{u}),(1-\gamma) d(\mathfrak{u}, \mathscr{M} \mathfrak{u}),(1-\gamma) d($ $\mathfrak{u}, \mathscr{M} \mathfrak{t}), \gamma d\left(\mathfrak{u}, \mathfrak{c}_{1}\right)+(1-\gamma) d\left(\mathfrak{c}_{1}, \mathscr{M} \mathfrak{u}\right),(1-\gamma) d\left(\mathfrak{u}, \mathfrak{c}_{1}\right)+\gamma d($ $\left.\left.\mathfrak{c}_{1}, \mathscr{M} \mathfrak{u}\right)\right\}=2 \mathfrak{a}$, then $\Theta(d(\mathfrak{u}, \mathscr{M} \mathfrak{u}))<\Theta(2 \mathfrak{a})$.

By definition of $\mathfrak{a}$ and $\Theta, \Theta(2 \mathfrak{a}) \leq \Theta(d(\mathfrak{t}, \mathscr{M} \mathfrak{a}))<\Theta(2 \mathfrak{a})$, a contradiction.

Case 2. If $\max \{2 \mathfrak{a}, \gamma d(\mathfrak{u}, \mathscr{M} \mathfrak{u}),(1-\gamma) d(\mathfrak{u}, \mathscr{M} \mathfrak{u}),(1-\gamma) d($ $\mathfrak{u}, \mathscr{M} \mathfrak{i}), \gamma d\left(\mathfrak{u}, \mathfrak{c}_{1}\right)+(1-\gamma) d\left(\mathfrak{c}_{1}, \mathscr{M} \mathfrak{t}\right),(1-\gamma) d\left(\mathfrak{u}, \mathfrak{c}_{1}\right)+\gamma d($ $\left.\left.\mathfrak{c}_{1}, \mathscr{M} \mathfrak{u}\right)\right\}=\gamma d(\mathfrak{u}, \mathscr{M} \mathfrak{i})$, then $\Theta(d(\mathfrak{u}, \mathscr{M} \mathfrak{u}))<\Theta(\gamma d(\mathfrak{u}, \mathscr{M}$ $\mathfrak{a t})$.

If $\gamma=0, \Theta(d(\mathfrak{t}, \mathscr{M} \mathfrak{t}))<\Theta(0)$, a contradiction.

If $\gamma \in(0,1), \Theta(d(\mathfrak{u}, \mathscr{M} \mathfrak{u}))<\Theta(\gamma d(\mathfrak{u}, \mathscr{M} \mathfrak{u}))<\Theta(d(\mathfrak{u}$, $\mathscr{M} \mathfrak{u})$ ), a contradiction.

Case 3. If $\max \{2 \mathfrak{a}, \gamma d(\mathfrak{u}, \mathscr{M} \mathfrak{u}),(1-\gamma) d(\mathfrak{u}, \mathscr{M} \mathfrak{u}),(1-\gamma) d($ $\mathfrak{u}, \mathscr{M} \mathfrak{u}), \gamma d\left(\mathfrak{u}, \mathfrak{c}_{1}\right)+(1-\gamma) d\left(\mathfrak{c}_{1}, \mathscr{M} \mathfrak{u}\right),(1-\gamma) d\left(\mathfrak{u}, \mathfrak{c}_{1}\right)+\gamma d($ $\left.\left.\mathfrak{c}_{1}, \mathscr{M} \mathfrak{i}\right)\right\}=(1-\gamma) d(\mathfrak{u}, \mathscr{M} \mathfrak{i})$, then $\Theta(d(\mathfrak{u}, \mathscr{M} \mathfrak{i}))<\Theta((1-$ $\gamma) d(\mathfrak{u}, \mathscr{M} \mathfrak{u})) \leq \Theta(d(\mathfrak{u}, \mathscr{M} \mathfrak{u}))$, a contradiction.

Case 4. If $\max \{2 \mathfrak{a}, \gamma d(\mathfrak{u}, \mathscr{M} \mathfrak{u}),(1-\gamma) d(\mathfrak{u}, \mathscr{M} \mathfrak{u}),(1-\gamma) d($ $\mathfrak{u}, \mathscr{M} \mathfrak{t}), \gamma d\left(\mathfrak{u}, \mathfrak{c}_{1}\right)+(1-\gamma) d\left(\mathfrak{c}_{1}, \mathscr{M} \mathfrak{u}\right),(1-\gamma) d\left(\mathfrak{u}, \mathfrak{c}_{1}\right)+\gamma d($ $\left.\left.\mathfrak{c}_{1}, \mathscr{M} \mathfrak{u}\right)\right\}=\gamma d\left(\mathfrak{u}, \mathfrak{c}_{1}\right)+(1-\gamma) d\left(\mathfrak{c}_{1}, \mathscr{M} \mathfrak{u}\right)$, then

$$
\begin{aligned}
\Theta(d(\mathfrak{u}, \mathscr{M} \mathfrak{u})) & <\Theta\left(\gamma d\left(\mathfrak{u}, \mathfrak{c}_{1}\right)+(1-\gamma) d\left(\mathfrak{c}_{1}, \mathscr{M} \mathfrak{u}\right)\right) \\
& <\Theta(\gamma \mathfrak{a}+(1-\gamma) \mathfrak{a})=\Theta(\mathfrak{a}) .
\end{aligned}
$$

By definition of $\mathfrak{a}$ and $\Theta, \Theta(2 \mathfrak{a}) \leq \Theta(d(\mathfrak{u}, \mathscr{M} \mathfrak{u}))<\Theta(\mathfrak{a})$, a contradiction.

Case 5. If $\max \{2 \mathfrak{a}, \gamma d(\mathfrak{u}, \mathscr{M} \mathfrak{u}),(1-\gamma) d(\mathfrak{u}, \mathscr{M} \mathfrak{u}),(1-\gamma) d($ $\mathfrak{u}, \mathscr{M} \mathfrak{i}), \gamma d\left(\mathfrak{u}, \mathfrak{c}_{1}\right)+(1-\gamma) d\left(\mathfrak{c}_{1}, \mathscr{M} \mathfrak{t}\right),(1-\gamma) d\left(\mathfrak{u}, \mathfrak{c}_{1}\right)+\gamma d($ $\left.\left.\mathfrak{c}_{1}, \mathscr{M} \mathfrak{u}\right)\right\}=(1-\gamma) d\left(\mathfrak{u}, \mathfrak{c}_{1}\right)+\gamma d\left(\mathfrak{c}_{1}, \mathscr{M} \mathfrak{u}\right)$, then

$$
\begin{aligned}
\Theta(d(\mathfrak{u}, \mathscr{M} \mathfrak{u})) & <\Theta\left((1-\gamma) d\left(\mathfrak{u}, \mathfrak{c}_{1}\right)+\gamma d\left(\mathfrak{c}_{1}, \mathscr{M} \mathfrak{u}\right)\right) \\
& <\Theta(\gamma \mathfrak{a}+(1-\gamma) \mathfrak{a})=\Theta(\mathfrak{a}) .
\end{aligned}
$$


By definition of $\mathfrak{a}$ and $\Theta, \Theta(2 \mathfrak{a}) \leq \Theta(d(\mathfrak{u}, \mathscr{M} \mathfrak{u}))<\Theta(\mathfrak{a})$, a contradiction.

Similarly, we can prove for $\mathfrak{c}_{2} \in \mathcal{U}$.

Hence, $\mathscr{M} \mathfrak{u}=\mathfrak{u}, \mathfrak{t} \in \mathscr{E}\left(\mathfrak{c}_{1}, \mathfrak{c}_{2}, \mathfrak{a}\right)$; that is, $\mathscr{E}\left(\mathfrak{c}_{1}, \mathfrak{c}_{2}, \mathfrak{a}\right)$ is a fixed ellipse of $\mathscr{M}$.

Theorem 16. If in the above theorem $d\left(\mathfrak{c}_{1}, \mathscr{M}_{\mathfrak{u}}\right)+d\left(\mathfrak{c}_{2}, \mathscr{M} \mathfrak{u}\right.$ )$\leq \mathfrak{a}$, then $\mathscr{E}_{\mathscr{D}}\left(\mathfrak{c}_{1}, \mathfrak{c}_{2}, \mathfrak{a}\right)$ is a fixed elliptic disc of $\mathscr{M}$.

Proof. Now, to show $\mathscr{E}_{\mathscr{D}}\left(\mathfrak{c}_{1}, \mathfrak{c}_{2}, \mathfrak{a}\right)$ is a fixed elliptic disc of $\mathscr{M}$, it is sufficient to demonstrate that $\mathscr{M}$ fixes an ellipse $\mathscr{E}\left(\mathfrak{c}_{1}\right.$, $\left.\mathfrak{c}_{2}, \mathfrak{b}\right)$ with $\mathfrak{b} \triangleleft \mathfrak{a}$. Since $\mathscr{M}$ is a generalized $\Theta_{\mathscr{C}}$-weak contraction, then proceeding as in Theorem $15, \mathscr{E}\left(\mathfrak{c}_{1}, \mathfrak{c}_{2}, \mathfrak{b}\right)$ is a fixed ellipse of $\mathscr{M}$ as $d\left(\mathfrak{c}_{1}, \mathscr{M} \mathfrak{u}\right)+d\left(\mathfrak{c}_{2}, \mathscr{M} \mathfrak{u}\right)=\mathfrak{b} \leq \mathfrak{a}$; that is, $\mathscr{M} \mathfrak{u}$ $=\mathfrak{u}, \forall \mathfrak{u} \in \mathscr{E}_{\mathscr{D}}\left(\mathfrak{c}_{1}, \mathfrak{c}_{2}, \mathfrak{b}\right)$. Hence, $\mathscr{E}_{\mathscr{D}}\left(\mathfrak{c}_{1}, \mathfrak{c}_{2}, \mathfrak{a}\right)$ is a fixed elliptic disc of $\mathscr{M}$.

Theorem 17. Theorem 15 remains true if we substitute Cirićtype $\Theta_{\mathscr{G}}$-weak contraction in place of generalized $\Theta_{\mathscr{g}}$-weak contraction.

Proof. The proof follows the pattern of Theorem 15 on taking $\gamma=0$.

Theorem 18. Theorem 16 remains true if we substitute Cirićtype $\Theta_{\mathscr{G}}$-weak contraction in place of generalized $\Theta_{\mathscr{G}}$-weak contraction.

Proof. The proof follows the pattern of Theorem 16 on taking $\gamma=0$.

The subsequent examples elucidate Theorems 17 and 18.

Example 4. Let $\mathscr{U}=[5, \infty)$ and a metric $d: \mathscr{U} \times \mathscr{U} \longrightarrow \mathbb{R}^{+}$be defined as $d(\mathfrak{u}, \mathfrak{b})=|\mathfrak{u}-\mathfrak{v}|$.

Let $\Theta(t)=e^{t}, \mathfrak{c}_{1}=-2, \mathfrak{c}_{2}=3, \mathfrak{a}=6, \gamma=0$, and $\alpha=6 / 7$.

The ellipse

$$
\begin{aligned}
\mathscr{E}(-2,3,6) & =\{\mathfrak{u} \in \mathscr{U}: d(-2, \mathfrak{u})+d(3, \mathfrak{u})=6\} \\
& =\{\mathfrak{u} \in \mathscr{U}:|-2-\mathfrak{u}|+|3-\mathfrak{u}|=6\}=\{-2.5,3.5\} .
\end{aligned}
$$

Define a self-map $\quad \mathscr{M}: \mathscr{U} \longrightarrow \mathscr{U}$ as $\mathscr{M} \mathfrak{u}=$ $\left(\begin{array}{ll}\mathfrak{t}, & \mathfrak{u} \in[-5,5] \\ \mathfrak{u}+12, & \text { otherwise }\end{array}\right.$.

Since for $\mathfrak{t} \in[-5,5], d(\mathfrak{u}, \mathscr{M} \mathfrak{t})=0$, and for $\mathfrak{u} \in(5, \infty)$, $d(\mathfrak{u}, \mathscr{M} \mathfrak{u})=12>0$.
Case 1. For $\mathfrak{u}>5$ and $\mathfrak{c}_{1}=-2$,

$$
\begin{aligned}
\mathscr{L}(\mathfrak{i t},-2) & =\max \{d(\mathfrak{i t},-2), d(-2, \mathscr{M}(-2)), d(\mathfrak{u}, \mathscr{M} \mathfrak{i t}), d(-2, \mathscr{M} \mathfrak{i t}), d(\mathfrak{u}, \mathscr{M}(-2))\} \\
& =\max \{d(\mathfrak{u},-2), d(-2,-2), d(\mathfrak{i t}, \mathscr{M} \mathfrak{i t}), d(-2, \mathscr{M} \mathfrak{i t}), d(\mathfrak{u},-2)\} \\
& =\max \{d(\mathfrak{t},-2), 0,12, d(-2, \mathfrak{t}+12)\}=\max \{|\mathfrak{u}+2|, 12,|\mathfrak{u}+14|\} \\
& =|\mathfrak{t}+14|>19,
\end{aligned}
$$

and $\quad \Theta(d(\mathfrak{u}, \mathscr{M} \mathfrak{u}))=\Theta(12)=e^{12}<e^{(12 / 13)|\mathfrak{u}+8|}=$ $e^{(\mathscr{L}(\mathfrak{u},-2))^{(12 / 19)}}=[\Theta(\mathscr{L}(\mathfrak{u t},-2))]^{(12 / 19)}$.

Case 2. For $\mathfrak{u}>5$ and $\mathfrak{c}_{2}=3$,

$$
\begin{aligned}
\mathscr{L}(\mathfrak{u}, 3) & =\max \{d(\mathfrak{u}, 3), d(3, \mathscr{M} 3), d(\mathfrak{u}, \mathscr{M} \mathfrak{u}), d(3, \mathscr{M} \mathfrak{u}), d(\mathfrak{u}, \mathscr{M} 3)\} \\
& =\max \{d(\mathfrak{u}, 3), d(3,3), d(\mathfrak{u}, \mathscr{M} \mathfrak{u}), d(3, \mathscr{M} \mathfrak{u}), d(\mathfrak{u}, 3)\} \\
& =\max \{d(\mathfrak{u}, 3), 0,12, d(3, \mathfrak{u}+12)\} \\
& =\max \{|\mathfrak{u}-3|, 12,|\mathfrak{u}+9|\}=|\mathfrak{u}+9|>14,
\end{aligned}
$$

and $\Theta\left(d\left(\mathfrak{u}, \mathscr{M}_{\mathfrak{u}}\right)\right)=\Theta(12)=e^{12}<e^{(12 / 14)|\mathfrak{u}+9|}=e^{(\mathscr{L}(\mathfrak{u}, 3))^{(12 / 14)}}$ $=[\Theta(\mathscr{L}(\mathfrak{t}, 3))]^{(12 / 14)}$.

That is, $\mathscr{M}$ is a Ćirić-type $\Theta_{\mathscr{G}}$-weak contraction with $\mathfrak{c}_{1}$ $=-2, \mathfrak{c}_{2}=3$, and $\alpha=\max \{12 / 19,12 / 14\}=12 / 14$. Hence, $\mathscr{E}$ $(-2,3,6)=\{-2.5,3.5\}$ is a fixed ellipse and $\mathscr{E}_{\mathscr{D}}(-2,3,6)=[$ $-2.5,3.5]$ is a fixed elliptic disc of $\mathscr{M}$. One may verify that $d$ $(-2, \mathfrak{u})+d(3, \mathfrak{u}) \leq 6, \mathfrak{u} \in \mathscr{C}_{\mathscr{D}}(-2,3,6)$.

Example 5. Let $\mathscr{U}=\mathbb{R}^{2}$ and a metric $d: \mathscr{U} \times \mathscr{U} \longrightarrow \mathbb{R}^{+}$be defined as $d(\mathfrak{u}, \mathfrak{v})=\sqrt{\left(\mathfrak{u}_{1}-\mathfrak{v}_{1}\right)^{2}+\left(\mathfrak{u}_{2}-\mathfrak{b}_{2}\right)^{2}}$, where $\mathfrak{u}=($ $\left.\mathfrak{u}_{1}, \mathfrak{u}_{2}\right)$ and $\mathfrak{v}=\left(\mathfrak{v}_{1}, \mathfrak{v}_{2}\right)$.

Let $\Theta(t)=1+t, \mathfrak{c}_{1}=(3+2 \sqrt{3},-1), \mathfrak{c}_{2}=(3-2 \sqrt{3},-1), \mathfrak{a}$ $=8, \gamma=0$, and $\alpha=6 / 7$.

The ellipse

$$
\begin{aligned}
\mathscr{E}\left(\mathfrak{c}_{1}, \mathfrak{c}_{2}, 8\right)= & \left\{\mathfrak{u} \in \mathscr{U}: d\left(\mathfrak{c}_{1}, \mathfrak{u}\right)+d\left(\mathfrak{c}_{2}, \mathfrak{u}\right)=8\right\} \\
= & \left\{\mathfrak{u} \in \mathcal{U}: \sqrt{\left(\mathfrak{u}_{1}-3-2 \sqrt{3}\right)^{2}+\left(\mathfrak{u}_{2}+1\right)^{2}}\right. \\
& \left.+\sqrt{\left(\mathfrak{u}_{1}-3+2 \sqrt{3}\right)^{2}+\left(\mathfrak{u}_{2}+1\right)^{2}}=8\right\} \\
= & \left\{\mathfrak{u} \in \mathcal{U}: \frac{\left(\mathfrak{u}_{1}-3\right)^{2}}{16}+\frac{\left(\mathfrak{u}_{2}+1\right)^{2}}{4}=1\right\},
\end{aligned}
$$

which is shown by the blue line in Figure 2 .

Further, the elliptic disc $\mathscr{E}_{\mathscr{D}}\left(\mathfrak{c}_{1}, \mathfrak{c}_{2}, 8\right)=\{\mathfrak{u} \in \mathscr{U}:($ $\left.\left.\left(\mathfrak{t}_{1}-3\right)^{2} / 16\right)+\left(\left(\mathfrak{t}_{2}+1\right)^{2} / 4\right) \leq 1\right\}$, which is shown as the blue shaded portion in Figure 2.

Define a self-map $\quad \mathscr{M}: \mathscr{U} \longrightarrow \mathscr{U}$ as $\mathscr{M} \mathfrak{u}=$

$$
\left(\begin{array}{ll}
\mathfrak{t}, & \mathfrak{u} \in(3+6 \cos \theta,-1+6 \sin \theta) \\
\mathfrak{u}+(8 \sqrt{2}, 8 \sqrt{2}), & \text { otherwise }
\end{array} .\right.
$$

Since for $\mathfrak{t} \in(3+6 \cos \theta,-1+6 \sin \theta), d(\mathfrak{u}, \mathscr{M} \mathfrak{t})=0$, and for $\mathfrak{u} \in \mathbb{R}^{2} \backslash(3+6 \cos \theta,-1+6 \sin \theta), \quad d(\mathfrak{u}, \mathscr{M} \mathfrak{u})=16$ $>0$. 
Case 1. For $\mathfrak{u} \in \mathbb{R}^{2} \backslash(3+6 \cos \theta,-1+6 \sin \theta)$ and $\mathfrak{c}_{1}=(3+$ $2 \sqrt{3},-1)$,

$$
\begin{aligned}
\mathscr{L}(\mathfrak{u},(3+2 \sqrt{3},-1))= & \max \{d(\mathfrak{u},(3+2 \sqrt{3},-1)), d \\
& \cdot((3+2 \sqrt{3},-1), \mathscr{M}(3+2 \sqrt{3},-1)), d \\
& \cdot(\mathfrak{u}, \mathscr{M} \mathfrak{u}), d((3+2 \sqrt{3},-1), \mathscr{M} \mathfrak{u}), d \\
& \cdot(\mathfrak{u}, \mathscr{M}(3+2 \sqrt{3},-1))\} \\
= & \max \{d(\mathfrak{u},(3+2 \sqrt{3},-1)), d \\
& \cdot((3+2 \sqrt{3},-1),(3+2 \sqrt{3},-1)), d \\
& \cdot(\mathfrak{u}, \mathscr{M} \mathfrak{u}), d((3+2 \sqrt{3},-1), \mathscr{M} \mathfrak{u}), d \\
& \cdot(\mathfrak{u},(3+2 \sqrt{3},-1))\} \\
= & \max \{d(\mathfrak{u},(3+2 \sqrt{3},-1)), 0,16, d \\
& \cdot((3+2 \sqrt{3},-1), \mathfrak{u}+(8 \sqrt{2}, 8 \sqrt{2}))\} \\
= & \max \left\{\sqrt{\left(\mathfrak{u}_{1}-3-2 \sqrt{3}\right)^{2}+\left(\mathfrak{u}_{2}+1\right)^{2}}, 16,\right. \\
& \left.\cdot \sqrt{ }\left(\mathfrak{u}_{1}+8 \sqrt{2}-3-2 \sqrt{3}\right)^{2}+\left(\mathfrak{u}_{2}+8 \sqrt{2}+1\right)^{2}\right\} \\
> & 16,
\end{aligned}
$$

and $\quad \Theta(d(\mathfrak{u}, \mathscr{M} \mathfrak{u}))=\Theta(16)=e^{16}<e^{(16 / 17) \mathscr{L}(\mathfrak{u},(3+2 \sqrt{3},-1)) \mid}=$ $[\Theta(\mathscr{L}(\mathfrak{u},(3+2 \sqrt{3},-1)))]^{(16 / 17)}$.

Case 2. For $\mathfrak{t} \in \mathbb{R}^{2} \backslash(3+6 \cos \theta,-1+6 \sin \theta)$ and $\mathfrak{c}_{1}=(3-$ $2 \sqrt{3},-1)$

$$
\begin{aligned}
\mathscr{L}(\mathfrak{u},(3-2 \sqrt{3},-1))= & \max \{d(\mathfrak{u},(3-2 \sqrt{3},-1)), d \\
& \cdot((3-2 \sqrt{3},-1), \mathscr{M}(3-2 \sqrt{3},-1)), d \\
& \cdot(\mathfrak{u}, \mathscr{M} \mathfrak{u}), d((3-2 \sqrt{3},-1), \mathscr{M} \mathfrak{u}), d \\
& \cdot(\mathfrak{u}, \mathscr{M}(3-2 \sqrt{3},-1))\} \\
= & \max \{d(\mathfrak{u},(3-2 \sqrt{3},-1)), d \\
& \cdot((3-2 \sqrt{3},-1),(3-2 \sqrt{3},-1)), d(\mathfrak{u}, \mathscr{M} \mathfrak{u}), d \\
& \cdot((3-2 \sqrt{3},-1), \mathscr{M} \mathfrak{u}), d(\mathfrak{u},(3-2 \sqrt{3},-1))\} \\
= & \max \{d(\mathfrak{u},(3-2 \sqrt{3},-1)), 0,16, d \\
& \cdot((3-2 \sqrt{3},-1), \mathfrak{u}+(8 \sqrt{2}, 8 \sqrt{2}))\} \\
= & \max \left\{\sqrt{\left(\mathfrak{u}_{1}-3+2 \sqrt{3}\right)^{2}+\left(\mathfrak{u}_{2}+1\right)^{2}, 16,}\right. \\
& \left.\cdot \sqrt{ }\left(\mathfrak{u}_{1}+8 \sqrt{2}-3+2 \sqrt{3}\right)^{2}+\left(\mathfrak{u}_{2}+8 \sqrt{2}+1\right)^{2}\right\}>21,
\end{aligned}
$$

$$
\begin{aligned}
\Theta(d(\mathfrak{u}, \mathscr{M} \mathfrak{u})) & =\Theta(16)=e^{16}<e^{(16 / 21) \mathscr{L}(\mathfrak{u},(3-2 \sqrt{3},-1))} \\
& =[\Theta(\mathscr{L}(\mathfrak{u},(3-2 \sqrt{3},-1)))]^{(16 / 21)} .
\end{aligned}
$$

That is, $\mathscr{M}$ is a Cirić-type $\Theta_{\mathscr{E}}$-weak contraction with $\mathfrak{c}_{1}$

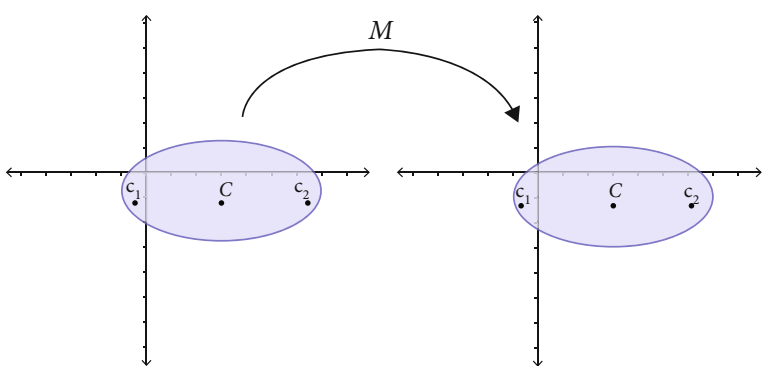

Figure 2: The blue line and its interior demonstrate the ellipse ( $\mathscr{E}$ $\left.\left(\mathfrak{c}_{1}, \mathfrak{c}_{2}, 8\right)\right)$ and elliptic disc $\left(\mathscr{E}_{\mathscr{D}}\left(\mathfrak{c}_{1}, \mathfrak{c}_{2}, 8\right)\right)$, respectively, which is fixed by map $\mathscr{M}$.

$=(3+2 \sqrt{3},-1), \mathfrak{c}_{2}=(3-2 \sqrt{3},-1)$, and $\alpha=\max \{16 / 17,16 /$ $21\}=16 / 17$. Hence, $\mathscr{E}\left(\mathfrak{c}_{1}, \mathfrak{c}_{2}, 8\right)$ is a fixed ellipse and $\mathscr{E}_{\mathscr{D}}\left(\mathfrak{c}_{1}\right.$ $\left., \mathfrak{c}_{2}, 8\right)$ is a fixed elliptic disc of $\mathscr{M}$. One may verify that $d\left(\mathfrak{c}_{1}\right.$ , $\mathfrak{t})+d\left(\mathfrak{c}_{2}, \mathfrak{u}\right) \leq 8, \mathfrak{t} \in \mathscr{E}_{\mathscr{D}}\left(\mathfrak{c}_{1}, \mathfrak{c}_{2}, 8\right)$.

Now, we introduce $\Psi_{\mathscr{g}}$-weak JS-contraction to study the geometry of nonunique fixed points.

Definition 19. Let $\Psi:[0, \infty) \longrightarrow[1, \infty)$ be an increasing function with $\Psi(0)=1$; then, a map $\mathscr{M}: \mathscr{U} \longrightarrow \mathscr{U}$ of a metric space $(\mathcal{U}, d)$ is said to be a $\Psi_{\mathscr{E}}$-weak JS-contraction with $\mathfrak{t}$ $\neq \mathfrak{v}$, if

$$
\begin{aligned}
d(\mathfrak{u}, \mathscr{M} \mathfrak{i})> & 0 \Rightarrow \Psi(d(\mathfrak{u}, \mathscr{M} \mathfrak{i})) \\
\leq & {[\Psi(d(\mathfrak{u}, \mathfrak{v}))]^{a}\left[\Psi\left(d\left(\mathfrak{u}, \mathscr{M}_{\mathfrak{u}}\right)\right)\right]^{b}[\Theta(d(\mathfrak{v}, \mathscr{M} \mathfrak{v}))]^{c} } \\
& \cdot[\Psi(d(\mathfrak{u}, \mathscr{M} \mathfrak{v}))]^{e}\left[\Psi\left(d\left(\mathfrak{v}, \mathscr{M}_{\mathfrak{u}}\right)\right)\right]^{f},
\end{aligned}
$$

where $a, b, c, e$, and $f$ are nonnegative and $a+b+c+e+f$ $\in[0,1), \mathfrak{u}, \mathfrak{v} \in \mathscr{U}$.

Theorem 20. Let $\mathscr{E}\left(\mathfrak{c}_{1}, \mathfrak{c}_{2}, \mathfrak{a}\right)$ be an ellipse in a metric space $(\mathscr{U}, d)$ and $\mathfrak{a}=(1 / 2)\{\inf d(\mathfrak{i t}, \mathscr{M} \mathfrak{i}): \mathfrak{i} \neq \mathscr{M} \mathfrak{i}\}$. If map $\mathscr{M}$ $: \mathcal{U} \longrightarrow \mathcal{U}$ is a $\Psi_{\text {or }}$-weak JS-contraction with $\mathfrak{c}_{1}, \mathfrak{c}_{2} \in \mathcal{U}$ and $d\left(\mathfrak{c}_{1}, \mathscr{M} \mathfrak{u}\right)+d\left(\mathfrak{c}_{2}, \mathscr{M} \mathfrak{u}\right)=\mathfrak{a}, \mathfrak{u} \in \mathscr{E}\left(\mathfrak{c}_{1}, \mathfrak{c}_{2}, \mathfrak{a}\right)$, then $\mathscr{E}\left(\mathfrak{c}_{1}, \mathfrak{c}_{2}, \mathfrak{a}\right.$ ) is a fixed ellipse of $\mathscr{M}$.

Proof. Let $\mathfrak{u} \in \mathscr{E}\left(\mathfrak{c}_{1}, \mathfrak{c}_{2}, \mathfrak{a}\right)$ be any arbitrary point and $\mathscr{M} \mathfrak{t} \neq \mathfrak{t}$ . From the definition of $\mathfrak{a}, d\left(\mathfrak{u}, \mathscr{M}_{\mathfrak{u}}\right) \geq 2 \mathfrak{a}$, since $\mathscr{M}$ is $\Psi_{\mathscr{E}}$ -weak JS-contraction for $\mathfrak{c}_{1}, \mathfrak{c}_{2} \in \mathcal{U}$, suppose $\mathscr{M} \mathfrak{c}_{1} \neq \mathfrak{c}_{1}$ and $\mathscr{M} \mathfrak{c}_{2} \neq \mathfrak{c}_{2}$, so we have $\left.\left.d\left(\mathfrak{c}_{1}, \mathscr{M} \mathfrak{c}_{1}\right)\right)>0, d\left(\mathfrak{c}_{2}, \mathscr{M} \mathfrak{c}_{2}\right)\right)>0$, and

$$
\begin{aligned}
\Psi\left(d\left(\mathfrak{c}_{1}, \mathscr{M} \mathfrak{c}_{1}\right)\right) \leq & {\left[\Psi\left(d\left(\mathfrak{c}_{1}, \mathfrak{c}_{1}\right)\right)\right]^{a}\left[\Psi\left(d\left(\mathfrak{c}_{1}, \mathscr{M} \mathfrak{c}_{1}\right)\right)\right]^{b}\left[\Psi\left(d\left(\mathfrak{c}_{1}, \mathscr{M} \mathfrak{c}_{1}\right)\right)\right]^{c} } \\
& \cdot\left[\Psi\left(d\left(\mathfrak{c}_{1}, \mathscr{M} \mathfrak{c}_{1}\right)\right)\right]^{e}\left[\Psi\left(d\left(\mathfrak{c}_{1}, \mathscr{M} \mathfrak{c}_{1}\right)\right)\right]^{f} \\
= & {[\Psi(0)]^{a}\left[\Psi\left(d\left(\mathfrak{c}_{1}, \mathscr{M} \mathfrak{c}_{1}\right)\right)\right]^{b+c+e+f} } \\
= & {\left[\Psi\left(d\left(\mathfrak{c}_{1}, \mathscr{M} \mathfrak{c}_{1}\right)\right)\right]^{1-a}<\Psi\left(d\left(\mathfrak{c}_{1}, \mathscr{M} \mathfrak{c}_{1}\right)\right), \text { a contradiction. } }
\end{aligned}
$$

So $\mathscr{M} \mathfrak{c}_{1}=\mathfrak{c}_{1}$. Similarly, $\mathscr{M} \mathfrak{c}_{2}=\mathfrak{c}_{2}$. 
Again, since $d(\mathfrak{u t}, \mathscr{M} \mathfrak{u})>0$, so

$$
\begin{aligned}
& \Psi(d(\mathfrak{u}, \mathscr{M} \mathfrak{u})) \leq {\left[\Psi\left(d\left(\mathfrak{u}, \mathfrak{c}_{1}\right)\right)\right]^{a}[\Psi(d(\mathfrak{u}, \mathscr{M} \mathfrak{u}))]^{b}\left[\Psi\left(d\left(\mathfrak{c}_{1}, \mathscr{M} \mathfrak{c}_{1}\right)\right)\right]^{c} } \\
& \cdot\left[\Psi\left(d\left(\mathfrak{u}, \mathscr{M} \mathfrak{c}_{1}\right)\right)\right]^{e}\left[\Psi\left(d\left(\mathfrak{c}_{1}, \mathscr{M} \mathfrak{u}\right)\right)\right]^{f} \\
&<< {[\Psi(\mathfrak{a})]^{a}[\Psi(2 \mathfrak{a})]^{b}\left[\Psi\left(d\left(\mathfrak{c}_{1}, \mathfrak{c}_{1}\right)\right)\right]^{c}[\Psi(\mathfrak{a})]^{e}[\Psi(\mathfrak{a})]^{f} } \\
&< {[\Theta(2 \mathfrak{a})]^{a}[\Psi(2 \mathfrak{a})]^{b}[\Theta(0)]^{c}[\Psi(2 \mathfrak{a})]^{e}[\Psi(2 \mathfrak{a})]^{f} } \\
& \cdot(\text { as } \Psi \text { is increasing })=[\Psi(2 \mathfrak{a})]^{a+b+e+f} \\
&<<(2 \mathfrak{a})]^{1-c}<\Psi(2 \mathfrak{a}) .
\end{aligned}
$$

Since $d(\mathfrak{t}, \mathscr{M} \mathfrak{i}) \geq 2 \mathfrak{a}$ and $\Psi$ is increasing, $\Psi(2 \mathfrak{a}) \leq \Psi(d($ $\mathfrak{u}, \mathscr{M} \mathfrak{u}))<\Psi(2 \mathfrak{a})$, a contradiction.

Similarly, we can prove for $\mathfrak{c}_{2} \in \mathcal{U}$.

Hence, $\mathscr{M} \mathfrak{t}=\mathfrak{t}, \mathfrak{t} \in \mathscr{E}\left(\mathfrak{c}_{1}, \mathfrak{c}_{2}, \mathfrak{a}\right)$; that is, $\mathscr{E}\left(\mathfrak{c}_{1}, \mathfrak{c}_{2}, \mathfrak{a}\right)$ is a fixed ellipse of $\mathscr{M}$.

Theorem 21. If in Theorem 20, $d\left(\mathfrak{c}_{1}, \mathscr{M} \mathfrak{i}\right)+d\left(\mathfrak{c}_{2}, \mathscr{M} \mathfrak{i}\right) \leq \mathfrak{a}$, then $\mathscr{E}_{\mathscr{D}}\left(\mathfrak{c}_{1}, \mathfrak{c}_{2}, \mathfrak{a}\right)$ is a fixed elliptic disc of $\mathscr{M}$.

Proof. The proof follows the pattern of Theorem 16 .

The subsequent example elucidates Theorems 20 and 21.

Example 6. Let $\mathcal{U}=\{-2,0,(1 / 2) \ln (6 / e),(1 / 2) \ln (15 / e),(1 /$ 2) $\ln (18 / e),(1 / 2) \ln (21 / e),(1 / 2) \ln (24 / e),(1 / 2) \ln (27 / e),($ $1 / 2) \ln (30 / e),(1 / 2) \ln (6 e),(1 / 2) \ln (9 e),(1 / 2) \ln (12 e),(1 /$ 2) $\ln (15 e), \ln 2, \ln 3, \ln 5\}$ and a metric $d: \mathscr{U} \times \mathscr{U} \longrightarrow \mathbb{R}^{+}$ be defined as $d(\mathfrak{u}, \mathfrak{v})=|\mathfrak{u}-\mathfrak{v}|$. Let $\Psi(t)=e^{t}, \mathfrak{c}_{1}=\ln 3, \mathfrak{c}_{2}=$ $\ln 5, \mathfrak{a}=1, \gamma=0$, and $\alpha=3 / 4$.

The ellipse

$$
\begin{aligned}
\mathscr{E}(\ln 3, \ln 5,1) & =\{\mathfrak{u} \in \mathcal{U}: d(\ln 3, \mathfrak{t})+d(\ln 5, \mathfrak{t})=1\} \\
& =\{\mathfrak{u} \in \mathcal{U}:|\ln 3-\mathfrak{t}|+|\ln 5-\mathfrak{t}|=1\} \\
& =\left\{\frac{1}{2} \ln \left(\frac{15}{e}\right), \frac{1}{2} \ln (15 e)\right\} .
\end{aligned}
$$

Define a self-map $\mathscr{M}: \mathscr{U} \longrightarrow \mathscr{U}$ as

$$
\mathscr{M}_{\mathfrak{t}}=\left(\begin{array}{ll}
0, & \mathfrak{u}=-2, \\
-2, & \mathfrak{t}=0, \\
\mathfrak{u}, & \text { otherwise }
\end{array}\right.
$$

Then, $d(\mathfrak{u}, \mathscr{M} \mathfrak{u})=\left(\begin{array}{ll}2, & \mathfrak{u} \in\{-2,0\} \\ 0, & \text { otherwise }\end{array}\right.$.

Then, $d(\mathfrak{u}, \mathscr{M} \mathfrak{u})=2>0$.
Case 1. For $\mathfrak{u}=\{-2,0\}$ and $\mathfrak{c}_{1}=\ln 3$,

$$
\begin{aligned}
& {[\Psi(d(\mathfrak{u}, \ln 3))]^{a}[\Psi(d(\mathfrak{u}, \mathscr{M} \mathfrak{i t}))]^{b}[\Psi(d(\ln 3, \mathscr{M} \ln 3))]^{c}} \\
& \times[\Psi(d(\mathfrak{u}, \mathscr{M} \ln 3))]^{e}[\Psi(d(\ln 3, \mathscr{M} \mathfrak{i}))]^{f} \\
& =[\Psi(|\mathfrak{t}-\ln 3|)]^{a}[\Psi(|\mathfrak{t}-\mathscr{M} \mathfrak{i t}|)]^{b}[\Psi(|\ln 3-\mathscr{M} \ln 3|)]^{c} \\
& \times[\Psi(|\mathfrak{t}-\mathscr{M} \ln 3|)]^{e}\left[\Psi\left(\left|\ln 3-\mathscr{M}_{\mathfrak{t}}\right|\right)\right]^{f} \\
& =[\Psi(|\mathfrak{u}-\ln 3|)]^{a}[\Psi(2)]^{b}[\Psi(|\ln 3-\ln 3|)]^{c}[\Psi(|\mathfrak{u}-\ln 3|)]^{e} \\
& \times[\Psi(|\ln 3-\mathscr{M} \mathfrak{u}|)]^{f} \\
& =[\Psi(|\mathfrak{t}-\ln 3|)]^{a+e}[\Psi(2)]^{b}[\Psi(|\ln 3-\mathscr{M} \mathfrak{u}|)]^{f} \\
& =[\Psi(|\mathfrak{u}-\ln 3|)]^{a+e}[\Psi(2)]^{b}[\Psi(|\ln 3-\mathscr{M} \mathfrak{i}|)]^{f},
\end{aligned}
$$

$$
\begin{aligned}
& {[\Psi(|\mathfrak{u}-\ln 3|)]^{a+e}[\Psi(2)]^{b}[\Psi(|\ln 3-\mathscr{M} \mathfrak{t}|)]^{f}} \\
& = \begin{cases}{[\Psi(\ln 3)]^{a+e}[\Psi(2)]^{b}[\Psi(|\ln 3+2|)]^{f},} & \text { if } \mathfrak{u}=0 \\
{[\Psi(|2+\ln 3|)]^{a+e}[\Psi(2)]^{b}[\Psi(\ln 3)]^{f},} & \text { if } \mathfrak{u}=-2\end{cases} \\
& = \begin{cases}{[\Psi(\ln 3)]^{a+e}[\Psi(2)]^{b}\left[\Psi\left(\ln \left(3 e^{2}\right)\right)\right],} & \text { if } \mathfrak{t}=0 \\
{\left[\Psi\left(\ln \left(3 e^{2}\right)\right)\right]^{a+e}[\Psi(2)]^{b}[\Psi(\ln 3)]^{f},} & \text { if } \mathfrak{t}=-2\end{cases} \\
& =\left\{\begin{array}{ll}
3^{a+e} e^{2 b}\left(3 e^{2}\right)^{f}, & \text { if } \mathfrak{u}=0 \\
\left(3 e^{2}\right)^{a+e} e^{2 b} 3^{f}, & \text { if } \mathfrak{u}=-2
\end{array}>e^{2}=\Psi(d(\mathfrak{u}, \mathscr{M} \mathfrak{u})),\right.
\end{aligned}
$$

for $a=e=1 / 4, b=1 / 4, c=0$, and $f=1 / 3$, satisfying $a+b+c$ $+e+f<1$; that is,

$$
\begin{aligned}
\Psi(d(\mathfrak{u}, \mathscr{M} \mathfrak{u}))< & {\left[\Psi\left(d\left(\mathfrak{c}_{1}, \mathfrak{c}_{1}\right)\right)\right]^{a}\left[\Psi\left(d\left(\mathfrak{c}_{1}, \mathscr{M} \mathfrak{c}_{1}\right)\right)\right]^{b}\left[\Psi\left(d\left(\mathfrak{c}_{1}, \mathscr{M} \mathfrak{c}_{1}\right)\right)\right]^{c} } \\
& \cdot\left[\Psi\left(d\left(\mathfrak{c}_{1}, \mathscr{M} \mathfrak{c}_{1}\right)\right)\right]^{e}\left[\Psi\left(d\left(\mathfrak{c}_{1}, \mathscr{M} \mathfrak{c}_{1}\right)\right)\right]^{f} .
\end{aligned}
$$

Case 2. For $\mathfrak{u} \in\{-2,0\}$ and $\mathfrak{c}_{2}=\ln 5$,

$$
\begin{aligned}
{[\Psi(} & d(\mathfrak{u}, \ln 5))]^{a}[\Psi(d(\mathfrak{u}, \mathscr{M} \mathfrak{t}))]^{b}[\Psi(d(\ln 5, \mathscr{M} \ln 5))]^{c} \\
& \times[\Psi(d(\mathfrak{u}, \mathscr{M} \ln 5))]^{e}[\Psi(d(\ln 5, \mathscr{M} \mathfrak{u}))]^{f} \\
= & {[\Psi(|\mathfrak{u}-\ln 5|)]^{a}[\Psi(|\mathfrak{u}-\mathscr{M} \mathfrak{u}|)]^{b}[\Psi(|\ln 5-\mathscr{M} \ln 5|)]^{c} } \\
& \times[\Psi(|\mathfrak{u}-\mathscr{M} \ln 5|)]^{e}[\Psi(|\ln 5-\mathscr{M} \mathfrak{u}|)]^{f} \\
= & {[\Psi(|\mathfrak{u}-\ln 5|)]^{a}[\Psi(2)]^{b}[\Psi(|\ln 5-\ln 5|)]^{c} } \\
& \times[\Psi(|\mathfrak{u}-\ln 5|)]^{e}[\Psi(|\ln 5-\mathscr{M} \mathfrak{u}|)]^{f} \\
= & {[\Psi(|\mathfrak{u}-\ln 5|)]^{a+e}[\Psi(2)]^{b}[\Psi(|\ln 5-\mathscr{M} \mathfrak{u}|)]^{f}, }
\end{aligned}
$$

$$
\begin{aligned}
& {[\Psi(|\mathfrak{u}-\ln 5|)]^{a+e}[\Psi(2)]^{b}\left[\Psi\left(\left|\ln 5-M_{\mathfrak{i}}\right|\right)\right]^{f}} \\
& = \begin{cases}{[\Psi(\ln 5)]^{a+e}[\Psi(2)]^{b}[\Psi(|\ln 5+2|)]^{f},} & \text { if } \mathfrak{t}=0 \\
{[\Psi(|2+\ln 5|)]^{a+e}[\Psi(2)]^{b}[\Psi(\ln 5)]^{f},} & \text { if } \mathfrak{u}=-2\end{cases} \\
& = \begin{cases}{[\Psi(\ln 5)]^{a+e}[\Psi(2)]^{b}\left[\Psi\left(\ln \left(5 e^{2}\right)\right)\right]^{f},} & \text { if } \mathfrak{t}=0 \\
{\left[\Psi\left(\ln \left(5 e^{2}\right)\right)\right]^{a+e}[\Psi(2)]^{b}[\Psi(\ln 5)]^{f},} & \text { if } \mathfrak{t}=-2\end{cases} \\
& =\left\{\begin{array}{ll}
5^{a+e} e^{2 b}\left(5 e^{2}\right)^{f}, & \text { if } \mathfrak{t}=0 \\
\left(5 e^{2}\right)^{a+e} e^{2 b} 5^{f}, & \text { if } \mathfrak{t}=2
\end{array}>e^{2}=\Psi(d(\mathfrak{u}, \mathscr{M} \mathfrak{u})),\right.
\end{aligned}
$$


for $a=e=1 / 4, b=1 / 4, c=0$, and $f=1 / 3$, satisfying $a+b+c$ $+e+f<1$; that is,

$$
\begin{aligned}
\Psi(d(\mathfrak{u}, \mathscr{M} \mathfrak{u}))< & {\left[\Psi\left(d\left(\mathfrak{c}_{1}, \mathfrak{c}_{1}\right)\right)\right]^{a}\left[\Psi\left(d\left(\mathfrak{c}_{1}, \mathscr{M} \mathfrak{c}_{1}\right)\right)\right]^{b} } \\
& \cdot\left[\Psi\left(d\left(\mathfrak{c}_{1}, \mathscr{M} \mathfrak{c}_{1}\right)\right)\right]^{c}\left[\Psi\left(d\left(\mathfrak{c}_{1}, \mathscr{M} \mathfrak{c}_{1}\right)\right)\right]^{e} \\
& \cdot\left[\Psi\left(d\left(\mathfrak{c}_{1}, \mathscr{M} \mathfrak{c}_{1}\right)\right)\right]^{f} .
\end{aligned}
$$

That is, $\mathscr{M}$ is a $\Psi$-weak JS-contraction with $\mathfrak{c}_{1}=\ln 3, \mathfrak{c}_{2}$ $=\ln 5$, and $a=e=1 / 4, b=1 / 4, c=0$, and $f=1 / 3$. Hence, $\mathscr{E}$ $(\ln 3, \ln 5,1)=\{(1 / 2) \ln (15 / e),(1 / 2) \ln (15 e)\}$ is a fixed ellipse and $\mathscr{E}_{\mathscr{D}}(\ln 3, \ln 5,1)=\mathscr{U} \backslash\{-2,0\}$ is a fixed elliptic disc of $\mathscr{M}$. One may verify that $d(\ln 3, \mathfrak{u})+d(\ln 5, \mathfrak{t}) \leq 1, \mathfrak{t}$ $\in \mathscr{E}_{\mathscr{D}}(\ln 3, \ln 5,1)$. Noticeably, a fixed ellipse or a fixed elliptic disc is not essentially unique, as $\mathscr{E}(\ln 3, \ln 5,1)$ is also a fixed ellipse and $\mathscr{E}_{\mathscr{D}}(\ln 3, \ln 5,1)$ is also a fixed elliptic disc of $\mathscr{M}$.

Now, we prove the fixed ellipse and fixed elliptic disc conclusions of the integra-type. Let $\Phi:[0, \infty) \longrightarrow[0, \infty)$ be a locally integrable function so that, for each $t>0, \int_{0}^{1} \Phi(t) d t$ $>0$.

Definition 22. Let $\Theta:(0, \infty) \longrightarrow(1, \infty)$ be an increasing function. A map $\mathscr{U}: \mathcal{U} \longrightarrow \mathscr{U}$ of a metric space $(\mathscr{U}, d)$ is said to be a generalized integral-type $\Theta_{\mathscr{E}}$-weak contraction with $\mathfrak{t} \neq \mathfrak{v}$, if

$$
d(\mathfrak{u}, \mathscr{M} \mathfrak{u})>0 \Rightarrow \int_{0}^{\Theta\left(d\left(\mathfrak{u}, \mathscr{M}_{\mathfrak{u}}\right)\right)} \Phi(t) d t \leq \int_{0}^{[\Theta(\mathscr{L}(\mathfrak{u}, \mathfrak{b}))]^{\alpha}} \Phi(t) d t
$$

where $\mathscr{L}(\mathfrak{u}, \mathfrak{v})=\max \{d(\mathfrak{u}, \mathfrak{v}), \gamma d(\mathfrak{u}, \mathscr{M} \mathfrak{u})+(1-\gamma) d(\mathfrak{v}, \mathscr{M}$ $\mathfrak{v}),(1-\gamma) d(\mathfrak{u}, \mathscr{M} \mathfrak{i})+\gamma d(\mathfrak{v}, \mathscr{M} \mathfrak{v}), \gamma d(\mathfrak{u}, \mathscr{M} \mathfrak{v})+(1-\gamma) d(\mathfrak{v}$, $\mathscr{M} \mathfrak{t}),(1-\gamma) d(\mathfrak{t}, \mathscr{M} \mathfrak{v})+\gamma d(\mathfrak{v}, \mathscr{M} \mathfrak{i t})\}, \gamma \in[0,1), \alpha \in(0,1), \mathfrak{t}$ , $\mathfrak{v} \in \mathcal{U}$.

Theorem 23. Let $\mathscr{E}\left(\mathfrak{c}_{1}, \mathfrak{c}_{2}, \mathfrak{a}\right)$ be an ellipse in a metric space $(\mathscr{U}, d)$ and $\mathfrak{a}=(1 / 2)\{\inf d(\mathfrak{u}, \mathscr{M} \mathfrak{i}): \mathfrak{u} \neq \mathscr{M} \mathfrak{u}\}$. If map $\mathscr{M}$ $: \mathcal{U} \longrightarrow \mathcal{U}$ is a generalized integral-type $\Theta_{\mathscr{E}}$-weak contraction with $\mathfrak{c}_{1}, \mathfrak{c}_{2} \in \mathscr{U}$ and $d\left(\mathfrak{c}_{1}, \mathscr{M} \mathfrak{i}\right)+d\left(\mathfrak{c}_{2}, \mathscr{M} \mathfrak{i}\right)=\mathfrak{a}, \mathfrak{u} \in \mathscr{E}\left(\mathfrak{c}_{1}, \mathfrak{c}_{2}\right.$, $\mathfrak{a})$, then $\mathscr{E}\left(\mathfrak{c}_{1}, \mathfrak{c}_{2}, \mathfrak{a}\right)$ is a fixed ellipse of $\mathscr{M}$.

Proof. The proof follows the pattern of Theorem 15 .

Theorem 24. If in Theorem 23, $d\left(\mathfrak{c}_{1}, \mathscr{M} \mathfrak{i}\right)+d\left(\mathfrak{c}_{2}, \mathscr{M} \mathfrak{i}\right) \leq \mathfrak{a}$, then $\mathscr{E}\left(\mathfrak{c}_{1}, \mathfrak{c}_{2}, \mathfrak{a}\right)$ is a fixed elliptic disc of $\mathscr{M}$.

Proof. The proof follows the pattern of Theorem 16 .

Next, we introduce an integral-type $\Psi_{\mathscr{E}}$-weak JScontraction.

Definition 25. Let $\Psi:[0, \infty) \longrightarrow[1, \infty)$ be an increasing function with $\Psi(0)=1$; then, map $\mathscr{M}: \mathscr{U} \longrightarrow \mathscr{U}$ of a metric space $(\mathcal{U}, d)$ is said to be an integral-type $\Psi_{\mathscr{E}}$-weak JS- contraction with $\mathfrak{u} \neq \mathfrak{v}$, if $d(\mathfrak{u}, \mathscr{M} \mathfrak{t})>0$ implies that

$$
\begin{aligned}
& \int_{0}^{\Psi(d(\mathfrak{u}, \mathscr{M} \mathfrak{u}))} \Phi(t) d t \\
& \quad \leq \int_{0}^{[\Psi(d(\mathfrak{u}, \mathfrak{b}))]^{\mathbf{a}}[\Psi(d(\mathfrak{u}, \mathscr{M} \mathfrak{u}))]^{\mathbf{b}}[\Theta(d(\mathfrak{v}, \mathscr{M} \mathfrak{v}))]^{\mathbf{c}}[\Psi(d(\mathfrak{u}, \mathscr{M} \mathfrak{v}))]^{\mathbf{e}}[\Psi(d(\mathfrak{v}, \mathscr{M} \mathfrak{u}))]^{\mathbf{f}}} \Phi(t) d t,
\end{aligned}
$$

where $\mathbf{a}, \mathbf{b}, \mathbf{c}, \mathbf{e}$, and $\mathbf{f}$ are nonnegative and $\mathbf{a}+\mathbf{b}+\mathbf{c}+\mathbf{e}+\mathbf{f}$ $\in[0,1), \mathfrak{t}, \mathfrak{v} \in \mathcal{U}$.

Theorem 26. Let $\mathscr{E}\left(\mathfrak{c}_{1}, \mathfrak{c}_{2}, \mathfrak{a}\right)$ be an ellipse in a metric space $(\mathscr{U}, d)$ and $\mathfrak{a}=(1 / 2)\{\inf d(\mathfrak{u}, \mathscr{M} \mathfrak{i}): \mathfrak{u} \neq \mathscr{M} \mathfrak{t}\}$. If map $\mathscr{M}$ $: \mathscr{U} \longrightarrow \mathcal{U}$ is an integral-type $\Psi_{\mathscr{E}}$-weak JS-contraction with $\mathfrak{c}_{1}, \mathfrak{c}_{2} \in \mathscr{U}$ and $d\left(\mathfrak{c}_{1}, \mathscr{M} \mathfrak{i}\right)+d\left(\mathfrak{c}_{2}, \mathscr{M} \mathfrak{v}\right)=\mathfrak{a}, \mathfrak{u} \in \mathscr{E}\left(\mathfrak{c}_{1}, \mathfrak{c}_{2}, \mathfrak{a}\right)$, then $\mathscr{E}\left(\mathfrak{c}_{1}, \mathfrak{c}_{2}, \mathfrak{a}\right)$ is a fixed ellipse of $\mathscr{M}$.

Proof. The proof follows the pattern of Theorem 15.

Theorem 27. If in Theorem 26, $d\left(\mathfrak{c}_{1}, \mathscr{M} \mathfrak{i t}\right)+d\left(\mathfrak{c}_{2}, \mathscr{M} \mathfrak{u}\right) \leq \mathfrak{a}$, then $\mathscr{E}\left(\mathfrak{c}_{1}, \mathfrak{c}_{2}, \mathfrak{a}\right)$ is a fixed elliptic disc of $\mathscr{M}$.

Proof. The proof follows the pattern of Theorem 16 .

\section{Remark 28.}

(1) It is interesting to mention here that if a self-map fixes an elliptic disc, then it also fixes an ellipse (see Examples 4, 5, and 6). However, the reverse may not be true

(2) The ellipses (elliptic discs) in metric spaces change their shapes on changing the center, lengths of a semiminor or semimajor axis, or metric under consideration. Also, it is not necessary that an ellipse or an elliptic disc defined in a metric space be the same as an ellipse or an elliptic disc in a Euclidean space. Noticeably, ellipses discussed in Examples 1, 4, and 6 and elliptic discs in Examples 4 and 6 are different from the ellipse and elliptic disc in a Euclidean space

(3) If there exists a self-map that maps the ellipse (elliptic disc) to itself, then that ellipse (elliptic disc) may not be a fixed ellipse (elliptic disc); that is, the self-map may not fix all the points of the ellipse (elliptic disc)

(4) All the elliptic discs inside a fixed elliptic disc are also fixed elliptic discs of a self-map. Further, a fixed ellipse is not essentially unique (see Example 6)

(5) If both the focuses coincide, then fixed ellipse results, as well as fixed elliptic disc results, reduce to analogous fixed circle and fixed disc results, respectively. Noticeably, if $\mathfrak{c}_{1}=\mathfrak{c}_{2}=\mathfrak{u}_{0}$ (say), then $\mathscr{E}\left(\mathfrak{c}_{1}, \mathfrak{c}_{2}, \mathfrak{a}\right)=$ $\mathscr{C}\left(\mathfrak{u}_{0}, \mathfrak{a} / 2\right)$ and $\mathscr{E}_{\mathscr{D}}\left(\mathfrak{c}_{1}, \mathfrak{c}_{2}, \mathfrak{a}\right)=\mathscr{D}\left(\mathfrak{u}_{0}, \mathfrak{a} / 2\right)$, with center $\mathfrak{t}_{0}$ and radius $\mathfrak{a} / 2$

(6) For work on the set of nonunique fixed points forming a fixed figure, one may refer to [1-23] and references therein 


\section{Applications}

Inspired by the fact that the fixed point theory is applicable in many real-world problems, we solve a boundary value problem arising when a diffusing matter is kept in an absorbing medium between parallel walls of fixed concentrations $\gamma$ and $\delta$. The concentration $\mathfrak{t}(t)$ of the substance at time $t$ is given by

$$
-\frac{d^{2} \mathfrak{u}}{d t^{2}}+\mathfrak{f}(t) \mathfrak{u}=\xi(t), \mathfrak{u}(0)=\gamma, \mathfrak{u}(1)=\delta,
$$

where $\mathfrak{f}(t)$ is the absorption coefficient and $\xi(t)$ is the source density.

The Green function associated with an initial value problem (48) is

$$
\mathscr{G}(t, s)=\left(\begin{array}{ll}
t(1-s), & 0 \leq t \leq s \leq 1, \\
s(1-t), & 0 \leq s \leq s \leq 1 .
\end{array}\right.
$$

Problem (48) is equivalent to

$$
\mathfrak{u}(t)=\gamma+(\delta-\gamma) t+\int_{0}^{1} \mathscr{G}(t, s)(\xi(s)-\mathfrak{f}(s) \mathfrak{u}(s)) d s, s \in[0,1] .
$$

Let $\mathcal{U}$ be a set of Riemann integrable functions on $[0,1]$; that is, $\mathscr{U}=R[0,1]$. Define $d: \mathscr{U} \times \mathscr{U} \longrightarrow \mathbb{R}^{+}$by

$$
d(\mathfrak{u}, \mathfrak{v})=\|\mathfrak{u}-\mathfrak{v}\|_{\infty}, \mathfrak{t}, \mathfrak{v} \in \mathscr{U},
$$

where $\|\mathfrak{u}\|_{\infty}=\sup _{t \in[0,1]}|\mathfrak{u}(t)|$. Clearly, $(\mathcal{U}, d)$ is a complete metric space.

Theorem 29. Consider the boundary value problem (48). Let $\mathscr{M}: \mathscr{U} \times \mathscr{U} \longrightarrow \mathbb{R}$ be a self-map in a complete metric space ( $\mathcal{U}, d)$, satisfying

$$
\begin{aligned}
\|\mathfrak{u}(t)-\mathfrak{v}(t)\|_{\infty} & >0 \Rightarrow\|\mathfrak{f}(t) \mathfrak{i t}(t)-\mathfrak{f}(t) \mathfrak{v}(t)\|_{\infty} \\
& \leq e^{-\lambda}\|\mathfrak{u}(t)-\mathfrak{v}(t)\|_{\infty}, \lambda>0 .
\end{aligned}
$$

Then, the boundary value problem (48) has a solution.

Proof. Define a map $\mathscr{M}: \mathscr{U} \longrightarrow \mathscr{U}$ by

$\mathscr{M} \mathfrak{u}(t)=\gamma+(\delta-\gamma) t+\int_{0}^{1} \mathscr{G}(t, s)(\xi(s)-\tilde{f}(s) \mathfrak{u}(s)) d s, s \in[0,1]$.

Clearly, a fixed point of map $\mathscr{M}$ is a solution to the problem (48).

$$
\begin{aligned}
\text { Since } \| \mathfrak{u}(t) & -\mathfrak{t}(t) \|_{\infty}>0, \\
d(\mathscr{M} \mathfrak{u}, \mathscr{M} \mathfrak{v})= & \mid \gamma+(\delta-\gamma) t+\int_{0}^{1} \mathscr{G}(t, s)(\xi(s)-\mathfrak{f}(s) \mathfrak{u}(s)) d s \\
& -\gamma-(\delta-\gamma) t-\int_{0}^{1} \mathscr{G}(t, s)(\xi(s)-\mathfrak{f}(s) \mathfrak{v}(s)) d s \mid \\
= & \mid \int_{0}^{1} \mathscr{G}(t, s)(\xi(s)-\mathfrak{f}(s) \mathfrak{u}(s)) d s \\
& -\int_{0}^{1} \mathscr{G}(t, s)(\xi(s)-\mathfrak{f}(s) \mathfrak{v}(s)) d s \mid \\
= & \left|\int_{0}^{1} \mathscr{G}(t, s)(\mathfrak{F}(s) \mathfrak{u}(s)-\mathfrak{f}(s) \mathfrak{v}(s)) d s\right| \\
< & \|\mathfrak{F}(s) \mathfrak{t}(s)-\mathfrak{f}(s) \mathfrak{v}(s)\|_{\infty} \sup _{t \in[0,1]}\left|\int_{0}^{1} \mathscr{G}(t, s) d s\right| \\
< & e^{-\lambda}\|\mathfrak{u}(s)-\mathfrak{v}(s)\|_{\infty} \sup _{t \in[0,1]}\left|\int_{0}^{1} \mathscr{G}(t, s) d s\right| \\
< & \frac{1}{8} e^{-\lambda}\|\mathfrak{u}(s)-\mathfrak{v}(s)\|_{\infty}=\frac{1}{8} e^{-\lambda} d(\mathfrak{u}, \mathfrak{v}) .
\end{aligned}
$$

If $\Theta(t)=e^{t}, t \in(0, \infty)$, then

$$
\begin{aligned}
\Theta(d(\mathscr{M} \mathfrak{u}, \mathscr{M} \mathfrak{v}))= & e^{d(\mathscr{M} \mathfrak{u}, \mathscr{U} \mathfrak{b})}<e^{(1 / 8) e^{-\lambda} d(\mathfrak{u}, \mathfrak{v})}=e^{d(\mathfrak{u}, \mathfrak{b})\left((1 / 1) e^{-\lambda}\right)} \\
= & {[\Theta(d(\mathfrak{u}, \mathfrak{v}))]^{\alpha} \leq[\Theta(\max \{d(\mathfrak{u}, \mathfrak{v}), d(\mathfrak{v}, \mathscr{M} \mathfrak{v}),} \\
& d(\mathfrak{u}, \mathscr{M} \mathfrak{u}), d(\mathfrak{v}, \mathscr{M} \mathfrak{u}), d(\mathfrak{u}, \mathscr{M} \mathfrak{v})]^{\alpha},
\end{aligned}
$$

where $\alpha=(1 / 8) e^{-\lambda}$ and $\alpha \in(0,1)$. Therefore, all the conditions of Theorem 8 are verified. Hence, $\mathscr{M}$ has a unique fixed point, which is indeed a unique solution to a boundary value problem (48).

\section{Conclusion}

We have explored new directions as a fixed ellipse and a fixed elliptic disc to the geometry of the set of nonunique fixed points of a map on a metric space via novel contractions. Further, we have utilized a generalized $\Theta$-contraction and Ćirićtype $\Theta$-contraction to establish a unique fixed point. Also, we have verified established results by illustrative examples to demonstrate the authenticity of the postulates and substantiated utility of our results by solving a boundary value problem of chemical sciences. The study of a set of unique and nonunique fixed points in the current context would be an interesting area for future study.

\section{Data Availability}

Data sharing is not applicable to this article as no datasets were generated or analyzed during the current study.

\section{Conflicts of Interest}

The authors declare no conflict of interest. 


\section{Authors' Contributions}

All authors contributed equally to this research.

\section{References}

[1] H. Aydi, N. Tas, N. Y. Özgür, and N. Mlaiki, "Fixed-discs in rectangular metric spaces," Symmetry, vol. 11, no. 2, p. 294, 2019.

[2] R. Bisht and N. Y. Özgür, "Geometric properties of discontinuous fixed point set of $(\varepsilon-\delta)$ contractions and applications to neural networks," Aequationes Math., vol. 94, no. 5, pp. 847863, 2019.

[3] M. Joshi, A. Tomar, and S. K. Padaliya, "Fixed point to fixed disc and application in partial metric spaces," in Chapter in a book "Fixed Point Theory and Its Applications to Real World Problem", Nova Science Publishers, New York, USA, 2021, ISBN: 978-1-53619-336-7.

[4] M. Joshi, A. Tomar, and S. K. Padaliya, "On geometric properties of non-unique fixed points in b-metric spaces," in Chapter in a book "Fixed Point Theory and Its Applications to Real World Problem", Nova Science Publishers, New York, USA, 2021, ISBN: 978-1-53619-336-7.

[5] M. Joshi, A. Tomar, and S. K. Padaliya, "Fixed point to fixed ellipse in metric spaces and discontinuous activation function," Applied Mathematics. E-Notes, vol. 1, p. 15, 2020.

[6] N. Mlaiki, N. Tas, and N. Y. Özgür, "On the fixed-circle problem and Khan type contractions," Axioms, vol. 7, no. 4, p. 80, 2018.

[7] N. Mlaiki, U. Çelik, N. Tas, N. Y. Özgür, and A. Mukheimer, "Wardowski type contractions and the fixed-circle problem on S-metric spaces," Journal of Mathematics, vol. 2018, Article ID 9127486, 9 pages, 2018.

[8] N. Mlaiki, N. Tas, and N. Y. Özgür, "New fixed-point theorems on an S-metric space via simulation functions," Mathematics, vol. 7, no. 7, p. 583, 2019.

[9] N. Mlaiki, N. Y. Özgür, and N. Tas, "New fixed-circle results related to Fc-contractive and Fc-expanding mappings on metric spaces," 2021, https://arxiv.org/abs/2101.10770.

[10] V. Ochkov, M. Nori, E. Borovinskaya, and W. Reschetilowski, "A new ellipse or math porcelain service," Symmetry, vol. 11, no. 2, p. 184, 2019.

[11] N. Y. Özgür, N. Tas, and U. Çelik, "Some fixed-circle results on S-metric spaces," Bulletin of Mathematical Analysis and Applications, vol. 9, no. 2, pp. 10-23, 2017.

[12] N. Y. Özgür and N. Tas, "Some fixed-circle theorems on metric spaces," Bulletin of the Malaysian Mathematical Sciences Society, vol. 42, no. 4, pp. 1433-1449, 2017.

[13] N. Y. Özgür and N. Tas, "Some fixed-circle theorems and discontinuity at fixed circle," in AIP Conference Proceedings, vol. 1926, article 020048, no. 1, 2018AIP Publishing LLC, 2018.

[14] N. Y. Özgür, "Fixed-disc results via simulation functions," Turkish Journal of Mathematics, vol. 43, no. 6, pp. 27942805, 2019.

[15] N. Y. Özgür and N. Tas, "Geometric properties of fixed points and simulation functions," 2021, https://arxiv.org/abs/2102 .05417 .

[16] R. P. Pant, N. Y. Özgür, and N. Tas, "On discontinuity problem at fixed point," Bulletin of the Malaysian Mathematical Sciences Society, vol. 43, no. 1, pp. 499-517, 2020.
[17] N. Tas, N. Y. Özgür, and N. Mlaiki, "New types of Fccontractions and the fixed-circle problem," Mathematics, vol. 6, no. 10, p. 188, 2018.

[18] N. Tas, "Bilateral-type solutions to the fixed-circle problem with rectified linear units application," Turkish Journal of Mathematics, vol. 44, no. 4, pp. 1330-1344, 2020.

[19] A. Tomar and M. Joshi, "Near fixed point, near fixed interval circle and near fixed interval disc in metric interval space," in Chapter in a book "Fixed Point Theory and Its Applications to Real World Problem", Nova Science Publishers, New York, USA, 2021, ISBN: 978-1-53619-336-7.

[20] A. Tomar, M. Joshi, and S. K. Padaliya, "Fixed point to fixed circle and activation function in partial metric space," Journal of Applied Analysis, vol. 1, 2020.

[21] A. Hussain, H. Al-Sulami, N. Hussain, and H. Farooq, "Newly fixed disc results using advanced contractions on F-metric space," Journal of Applied Analysis \& Computation, vol. 10, no. 6, pp. 2313-2322, 2020.

[22] M. Joshi, A. Tomar, H. A. Nabwey, and R. George, "On unique and nonunique fixed points and fixed circles in $\mathscr{M}_{v}^{b}$-metric space and application to cantilever beam problem," Journal of Function Spaces, vol. 2021, Article ID 6681044, 15 pages, 2021.

[23] H. N. Saleh, S. Sessa, W. M. Alfaqih, M. Imdad, and N. Mlaiki, "Fixed circle and fixed disc results for new types of $\Theta c$-contractive mappings in metric spaces," Symmetry, vol. 12, no. 11 , p. $1825,2020$.

[24] M. Fréchet, Sur quelques points du calcul fonctionnel, palemo (30 via Ruggiero), 1906.

[25] M. Jleli and B. Samet, "A new generalization of the Banach contraction principle," Journal of inequalities and applications, vol. 2014, 8 pages, 2014.

[26] L. B. Ćirić, "Generalised contractions and fixed-point theorems," Publications de l'Institut Mathématique, vol. 12, no. 26, pp. 9-26, 1971.

[27] S. Banach, "Sur les opérations dans les ensembles abstraits et leur application aux équations intégrales," Fundamenta Mathematicae, vol. 3, pp. 133-181, 1922. 